\title{
Beyond the standard seesaw: neutrino masses from Kähler operators and broken supersymmetry
}

\author{
Andrea Brignole, ${ }^{a}$ Filipe R. Joaquim $^{b, 1}$ and Anna Rossi ${ }^{c}$ \\ a INFN, Sezione di Padova, \\ I-35131 Padua, Italy \\ ${ }^{b}$ CERN, Theory Division, \\ CH-1211 Geneva 23, Switzerland \\ ${ }^{c}$ Dipartimento di Fisica "G. Galilei", Università di Padova, \\ I-35131 Padua, Italy \\ E-mail: andrea.brignole@pd.infn.it, Filipe.Joaquim@cern.ch, \\ arossi@pd.infn.it
}

ABSTRACT: We investigate supersymmetric scenarios in which neutrino masses are generated by effective $d=6$ operators in the Kähler potential, rather than by the standard $d=5$ superpotential operator. First, we discuss some general features of such effective operators, also including SUSY-breaking insertions, and compute the relevant renormalization group equations. Contributions to neutrino masses arise at low energy both at the tree level and through finite threshold corrections. In the second part we present simple explicit realizations in which those Kähler operators arise by integrating out heavy $\mathrm{SU}(2)_{W}$ triplets, as in the type II seesaw. Distinct scenarios emerge, depending on the mechanism and the scale of SUSY-breaking mediation. In particular, we propose an appealing and economical picture in which the heavy seesaw mediators are also messengers of SUSY breaking. In this case, strong correlations exist among neutrino parameters, sparticle and Higgs masses, as well as lepton flavour violating processes. Hence, this scenario can be tested at highenergy colliders, such as the LHC, and at lower energy experiments that measure neutrino parameters or search for rare lepton decays.

Keywords: Supersymmetry Breaking, Neutrino Physics, Supersymmetric Standard Model, Rare Decays

ARXIV EPRINT: 1007.1942

\footnotetext{
${ }^{1}$ On leave from the "Centro de Física Teórica de Partículas (CFTP)", Lisbon, Portugal.
} 


\section{Contents}

1 Introduction $\quad 1$

2 Neutrino masses from Kähler operators 3

2.1 Lepton number violating $d=6$ operators 3

2.2 Kähler operators $\left(H_{1}^{\dagger} L\right)^{2} / M^{2}$ with broken SUSY 4

2.3 Renormalization group evolution 5

$\begin{array}{lll}2.4 & \text { Tree-level contributions to neutrino masses } & 7\end{array}$

$\begin{array}{lll}2.5 & \text { Radiative finite contributions to } \mathbf{m}_{\nu} & 7\end{array}$

3 Type II seesaw realizations $\quad 9$

3.1 Type II in the SUSY limit $\quad 9$

$\begin{array}{ll}3.2 & \text { Type II with broken SUSY }\end{array}$

4 Seesaw mediators as SUSY-breaking messengers $\quad 12$

$\begin{array}{lll}4.1 & \text { SUSY-breaking mediation } & 12\end{array}$

$\begin{array}{lll}4.2 & \text { Neutrino masses } & 14\end{array}$

4.3 Phenomenological viability, MSSM spectrum and LHC searches 16

$\begin{array}{lll}4.4 & \text { Lepton flavour violation } & 19\end{array}$

5 Conclusions $\quad 22$

\section{Introduction}

The seesaw mechanism can be regarded as a paradigm to explain the smallness of neutrino masses. In the simplest scenarios, neutrinos acquire Majorana masses scaling as $m_{\nu} \sim$ $v^{2} / M$, where $v$ is the electroweak scale and $M \gg v$ is a heavy mass. The experimental neutrino data [1-13] point towards a natural value $M \sim 10^{15} \mathrm{GeV}$, close to the Grand Unification scale. From a low-energy perspective, the $1 / M$ dependence appears as the coefficient of the lowest dimension $(d=5) \mathrm{SU}(2)_{W} \times \mathrm{U}(1)_{Y}$ invariant operator which violates lepton number by two units $(\Delta L=2)$, namely $(H L)^{2} / M$, where $H$ and $L$ are Higgs and lepton doublets [14]. From a more fundamental perspective, this effective operator usually arises from integrating out heavy states with mass $\sim M$. At the tree level, such heavy seesaw mediators can be either singlet 'neutrinos' coupled to $H L$ (type I [15-20]), $\mathrm{SU}(2)_{W}$ triplet scalars with non-zero hypercharge coupled to $L L$ and $H H$ (type II [21-24]) or $\mathrm{SU}(2)_{W}$ triplet fermions with zero hypercharge coupled to $H L$ (type III $[25,26]$ ). These realizations can be also implemented in supersymmetric (SUSY) extensions of the Standard Model (SM). In such models, which contain two Higgs superfields ${ }^{1} H_{1}$ and $H_{2}$ with opposite

\footnotetext{
${ }^{1}$ Following the standard notation, we will use the same symbol for a Higgs (matter) chiral superfield and its scalar (fermionic) component field.
} 
hypercharges, the leading $\Delta L=2$ effective operator is the $d=5$ superpotential operator $\int d^{2} \theta\left(H_{2} L\right)^{2} / M$.

The scaling of neutrino masses with $v^{2} / M$ is not the only possibility, though. It is also conceivable that neutrino masses are suppressed by a higher power of the heavy scale $M$, the simplest possibility beyond $1 / M$ being

$$
m_{\nu} \sim \frac{m v^{2}}{M^{2}}
$$

where $m \ll M$ is another mass parameter. In fact, the non-SUSY type II seesaw [21-24] generically leads to neutrino masses depending on two mass parameters, like in eq. (1.1). The same occurs in suitable variants of the type I seesaw (see e.g. [27, 28]) or in some radiative mechanisms $[29,30]$. In other cases, $m_{\nu}$ is suppressed by even higher powers of $M$ (see e.g. [31-34]).

In this work, we will focus on SUSY models where neutrino masses behave like in eq. (1.1), with the additional requirement that $m$ is related to the electroweak scale. Consequently, $M$ is naturally lowered to intermediate values $M \lesssim 10^{9} \mathrm{GeV}$. The behaviour described by eq. (1.1) can be realized in various ways, through either Kähler or superpotential $d=6, \Delta L=2$ effective operators. In the latter case, a possible operator is $\int d^{2} \theta S\left(H_{2} L\right)^{2} / M^{2}$, where $S$ is a SM singlet with $\langle S\rangle \sim v$. This fits naturally in the framework of the next-to-minimal SUSY SM (NMSSM) [35], for which some tree-level realizations have been proposed in [36]. Regarding $d=6$ Kähler operators, two candidates have been pointed out in [37, 38], namely $\int d^{4} \theta\left(H_{1}^{\dagger} L\right)\left(H_{2} L\right) / M^{2}$ and $\int d^{4} \theta\left(H_{1}^{\dagger} L\right)^{2} / M^{2}$. In this case, neutrino masses arise in the form of (1.1) with $m \sim \mu$, where the superpotential parameter $\mu$ emerges from the replacement $F_{H_{1}}^{\dagger} \rightarrow-\mu H_{2}$.

The purpose of this article is to generalize the proposal of $[37,38]$ in several directions. In section 2 we describe some general features of $d=6, \Delta L=2$ effective operators and then focus on the Kähler operator $\left(H_{1}^{\dagger} L\right)^{2}$. We point out the importance of including SUSYbreaking insertions and find novel contributions to neutrino masses of the form (1.1), in which $m$ is a SUSY-breaking mass. Such SUSY-breaking contributions can be even the dominant source of neutrino masses. We also discuss and evaluate two classes of quantum effects related to those operators: low-energy finite corrections at the sparticle threshold and logarithmic corrections above it, described by renormalization group equations (RGEs). In section 3 we move from the effective level to a more fundamental one and present the simplest explicit realization of the Kähler operator $\left(H_{1}^{\dagger} L\right)^{2}$, including SUSY-breaking effects. This ultra-violet (UV) completion of the effective theory is obtained in a type II seesaw framework. The SUSY-breaking parameters associated with $\left(H_{1}^{\dagger} L\right)^{2}$ are related to those of the heavy triplet states in the case SUSY-breaking mediation occurs at or above the triplet scale, otherwise they can be generated radiatively, e.g. by low-scale gauge mediation and RGEs.

Another interesting feature of the SUSY type II seesaw is that it provides the simplest realization of minimal lepton flavour violation (LFV), in the sense that the high and lowenergy flavour structures are directly related [39]. In section 4 we present an appealing and predictive version of the type II seesaw where the heavy triplets, which generate 

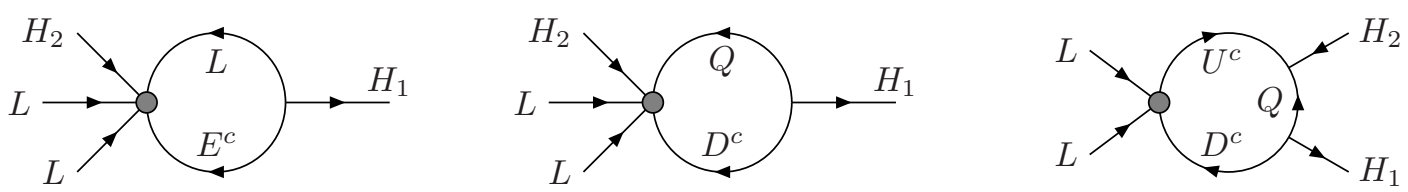

Figure 1. Logarithmically divergent contributions to the Kähler operator $\left(H_{1}^{\dagger} L\right)\left(H_{2} L\right) / M^{2}$ induced by other $d=6$ operators.

the $\Delta L=2$ effective operators at the tree level, are identified with the SUSY-breaking mediators, responsible for generating sparticle masses at the quantum level through gauge and Yukawa interactions. This scenario is a variant of that proposed in [40,41] and relates neutrino and sparticle masses even more closely, since their common source is the SUSYbreaking holomorphic mass term of the heavy states. In particular, we compute the full set of MSSM SUSY-breaking terms at the heavy triplet mass scale (section 4.1) and obtain the tree-level and quantum contributions to the neutrino mass matrix (section 4.2). We also discuss the phenomenological viability of this scenario, the general properties of the MSSM spectrum and the prospects for searches at the CERN Large Hadron Collider (LHC) (section 4.3). Special emphasis is devoted to LFV effects, which distinguish our model from purely gauge-mediated ones. In particular, we discuss the predictions for charged-lepton radiative decays $\ell_{i} \rightarrow \ell_{j} \gamma$, taking into account the near-future experimental sensitivity for both such LFV searches and the measurements of neutrino parameters (section 4.4). Finally, in section 5 we summarise our results and draw the concluding remarks.

\section{Neutrino masses from Kähler operators}

As anticipated in the Introduction, we are interested in SUSY scenarios in which neutrino masses are generated by effective $d=6, \Delta L=2$ operators. Before focussing on a specific class of such operators, let us briefly describe some of their general features.

\subsection{Lepton number violating $d=6$ operators}

Consider an effective low-energy theory with the field content of the minimal SUSY SM (MSSM) $[42,43]$ and conserved R-parity. The leading $\Delta L=2$ operator is the well-known $d=5$ term $\left(H_{2} L\right)^{2} / M \subset W$. In case this operator is (for some reason) suppressed or absent, there are two $d=6, \Delta L=2$ Kähler operators which can generate neutrino masses, namely $\left(H_{1}^{\dagger} L\right)\left(H_{2} L\right) / M^{2} \subset K$ and $\left(H_{1}^{\dagger} L\right)^{2} / M^{2} \subset K[37,38]$. We remark that, in principle, additional $d=6, \Delta L=2$ operators of the form $L L L E^{c} H_{2} / M^{2} \subset W, L L Q D^{c} H_{2} / M^{2} \subset W$ and $L L U^{c \dagger} D^{c} / M^{2} \subset K$ should be considered as well. Indeed, although these do not generate neutrino masses at the tree level, they do so radiatively, by inducing $\left(H_{1}^{\dagger} L\right)\left(H_{2} L\right) / M^{2} \subset K$ via RGEs (see figure 1). The fact that some operators mix under renormalization suggests that all the above $\Delta L=2$ operators could be grouped in distinct classes, by means of continuous or discrete Peccei-Quinn (PQ)-like symmetries under which $H_{1}^{\dagger}$ and $H_{2}$ transform differently and the ordinary Yukawa couplings are invariant. Since $\mu H_{2} H_{1} \subset W$ breaks 
such symmetries, we can consider the small $\mu$ parameter $(\mu \ll M)$ as a 'minimal' effective PQ spurion, such that non-invariant operators in $K$ and $W$ will be suppressed by powers of $\epsilon=\mu / \Lambda_{*}$, where $\Lambda_{*} \geq M$ is some high scale (e.g., that where $\mu$ is generated). For instance, if $\left(H_{1}^{\dagger} L\right)^{2} / M^{2} \subset K$ is allowed, we expect the remaining operators to be subleading, since the set $\left\{\left(H_{1}^{\dagger} L\right)\left(H_{2} L\right) / M^{2}, L L L E^{c} H_{2} / M^{2}, L L Q D^{c} H_{2} / M^{2}, L L U^{c \dagger} D^{c} / M^{2}\right\}$ will be suppressed by a factor $\epsilon$ while $\left(H_{2} L\right)^{2} / M$ will be suppressed by $\epsilon^{2}$. Alternatively, the symmetries may allow the operators $\left\{\left(H_{1}^{\dagger} L\right)\left(H_{2} L\right) / M^{2}, L L L E^{c} H_{2} / M^{2}, L L Q D^{c} H_{2} / M^{2}, L L U^{c \dagger} D^{c} / M^{2}\right\}$ and suppress the others.

Similar arguments can be applied to extensions of the MSSM in which $\mu$ is effectively generated at low energies by the vacuum expectation value (VEV) of a SM singlet $S$, through the term $S_{2} H_{1} \subset W$. The field $S$ is usually charged under some symmetry, such as a $Z_{3}$ in the NMSSM or an extra gauged U(1). Such symmetries provide selection rules for the effective operators as well. For instance, in the NMSSM framework $Z_{3}$ selection rules were used in [36] to generate neutrino masses at leading order through either $\left(H_{2} L\right)^{2} / M \subset W(d=5), S\left(H_{2} L\right)^{2} / M^{2} \subset W(d=6)$ or $S^{2}\left(H_{2} L\right)^{2} / M^{3} \subset W(d=7)$. Notice that only one of these operators can be invariant since each of them carries a different $Z_{3}$ charge. Nevertheless, if the allowed operator of this list has $d>5$, other $\Delta L=2$ operators are also allowed in $W$ or $K$ with the same or lower dimensionality, which were not considered in [36]. As an example, suppose $S\left(H_{2} L\right)^{2} / M^{2} \subset W$ is allowed by $Z_{3}$. Then, also the $d=6$ set $\left\{\left(H_{1}^{\dagger} L\right)\left(H_{2} L\right) / M^{2}, L L L E^{c} H_{2} / M^{2}, L L Q D^{c} H_{2} / M^{2}, L L U^{c \dagger} D^{c} / M^{2}\right\}$ is allowed. Alternatively, if $S^{2}\left(H_{2} L\right)^{2} / M^{3} \subset W$ is $Z_{3}$ symmetric, then several other operators of the same dimension $(d=7)$ such as $S\left(H_{1}^{\dagger} L\right)\left(H_{2} L\right) / M^{3}, S^{\dagger}\left(H_{2} L\right)^{2} / M^{3}, S L L U^{c \dagger} D^{c} / M^{2} \subset K$ or $S L L L E^{c} H_{2} / M^{3}, S L L Q D^{c} H_{2} / M^{3}, H_{1} H_{2}\left(H_{2} L\right)^{2} / M^{3} \subset W$ are permitted. Even more importantly, in this case there is a single $Z_{3}$-invariant operator of lower dimension, namely the $d=6$ term $\left(H_{1}^{\dagger} L\right)^{2} / M^{2} \subset K$.

The above discussion (which extends those of [36-38]) emphasizes the fact that symmetry arguments in the effective theory can partly justify the assumption that a specific operator dominates over others. The ultimate motivation for such a selection should lie at a more fundamental level, i.e., in the UV completion of the effective theory. From a minimal low-energy perspective, we note that the $d=6$ term $\left(H_{1}^{\dagger} L\right)^{2} / M^{2} \subset K$ is somehow singled out in the above examples by its symmetry properties. Furthermore, in section 3 we will show that this operator admits a very simple tree-level realization.

\subsection{Kähler operators $\left(H_{1}^{\dagger} L\right)^{2} / M^{2}$ with broken SUSY}

We proceed with our discussion by assuming that the leading $\Delta L=2$ effective operator has the form $\left(H_{1}^{\dagger} L\right)^{2} / M^{2}$. In general, we expect it to be accompanied by analogous operators with SUSY-breaking insertions ${ }^{2}$ of the form $X / M_{S}, X^{\dagger} / M_{S}, X X^{\dagger} / M_{S}^{2}$, where $X=\theta^{2} F_{X}$ is a SUSY-breaking spurion superfield (VEVs are understood) and $M_{S}$ is the scale of SUSY-

\footnotetext{
${ }^{2}$ Effective operators with $d>4$ and SUSY-breaking insertions have been also considered in other contexts, such as the Higgs sector [44-48] or baryon number violation [49-52]. SUSY-breaking effects in the neutrino sector have been considered in [53-59] from a perspective which is different from ours. In those works, SUSY breaking was invoked to suppress either $\mathrm{LH}_{2} N$ Yukawa couplings or $M_{N} N N$ mass terms (or both) in models with singlet states $N$.
} 
breaking mediation, which could be either larger or smaller than $M$. It is also tempting to identify $M_{S}$ with $M$, as we will do in section 4 .

In general, we can write the relevant $\Delta L=2$ effective lagrangian as

$$
\mathcal{L}_{\text {eff }}=\int d^{4} \theta \frac{1}{2 M^{2}}\left(\boldsymbol{\kappa}+\boldsymbol{\beta}_{\kappa} \frac{X}{M_{S}}+\tilde{\boldsymbol{\beta}}_{\kappa} \frac{X^{\dagger}}{M_{S}}+\boldsymbol{\gamma}_{\kappa} \frac{X X^{\dagger}}{M_{S}^{2}}\right)_{i j}\left(H_{1}^{\dagger} L_{i}\right)\left(H_{1}^{\dagger} L_{j}\right)+\text { h.c. }
$$

where $i, j=e, \mu, \tau$ are flavour indices, $\boldsymbol{\kappa}, \boldsymbol{\beta}_{\kappa}, \tilde{\boldsymbol{\beta}}_{\kappa}, \boldsymbol{\gamma}_{\kappa}$ are dimensionless flavour-dependent parameters and the SUSY gauge completion $\left(H_{1}^{\dagger} L\right) \rightarrow\left(H_{1}^{\dagger} e^{2 V} L\right)$ is understood. In principle, we could have incorporated the factor $1 / M^{2}$ into dimensionful coefficients as, for instance, $\boldsymbol{\kappa}^{\prime}=\boldsymbol{\kappa} / M^{2}$. This would better suit models in which the masses of the heavy states to be integrated out carry a flavour structure. However, even in such cases one can always factor out an overall $1 / M^{2}$. We have chosen the parametrization (2.1) to exhibit mass dimensions in a more transparent way, and also because the explicit realizations presented in sections 3 and 4 make use of heavy states with unflavoured masses.

By replacing $X$ with its SUSY-breaking VEV in eq. (2.1), we obtain the equivalent parametrization

$$
\mathcal{L}_{\text {eff }}=\int d^{4} \theta \frac{1}{2 M^{2}}\left(\boldsymbol{\kappa}+\theta^{2} \mathbf{B}_{\kappa}+\bar{\theta}^{2} \tilde{\mathbf{B}}_{\kappa}+\theta^{2} \bar{\theta}^{2} \mathbf{C}_{\kappa}\right)_{i j}\left(H_{1}^{\dagger} L_{i}\right)\left(H_{1}^{\dagger} L_{j}\right)+\text { h.c. },
$$

where we have traded the coefficients $\boldsymbol{\beta}_{\kappa}, \tilde{\boldsymbol{\beta}}_{\kappa}$ and $\boldsymbol{\gamma}_{\kappa}$ for dimensionful SUSY-breaking parameters $\mathbf{B}_{\kappa}=\boldsymbol{\beta}_{\kappa} F_{X} / M_{S}, \tilde{\mathbf{B}}_{\kappa}=\tilde{\boldsymbol{\beta}}_{\kappa} F_{X}^{*} / M_{S}$ (both of dimension one) and $\mathbf{C}_{\kappa}=\boldsymbol{\gamma}_{\kappa}\left|F_{X}\right|^{2} / M_{S}^{2}$ (of dimension two), respectively. The magnitude and flavour structure of all these parameters depend on the underlying physics which generates them. In sections 3 and 4 we will show explicit realizations which lead to simple correlations among the above quantities. Here, we will keep our discussion at a general and model-independent level. Notice that the SUSY part of $\mathcal{L}_{\text {eff }}$ is generated at $M$, while the SUSY-breaking one emerges at scales below $\min \left(M, M_{S}\right)$. At low energy, all four operators in eq. (2.1) [or, equivalently, in eq. (2.2)] contribute to neutrino masses either directly or indirectly, as we will show later. Before doing that, we will discuss the connection between high and low energies, namely the renormalization group evolution of the effective operators.

\subsection{Renormalization group evolution}

A convenient tool to derive the RGEs for the $\Delta L=2$ operators shown in eq. (2.2) is the general expression of the one-loop corrected Kähler potential obtained in [60], which applies to general effective SUSY theories with Kähler potential $K\left(\phi, \phi^{*}\right)$, superpotential $W(\phi)$ and gauge kinetic function $f_{a b}(\phi)$. The logarithmically divergent correction to $K$ reads [60]:

$$
(\Delta K)_{\log }=\frac{\log \Lambda_{\mathrm{UV}}^{2}}{32 \pi^{2}}\left[W_{i j} K^{j \bar{m}} W_{\bar{m} \bar{n}}^{*} K^{i \bar{n}}-4\left(\operatorname{Re} f_{a}\right)^{-1}\left(\phi^{\dagger} T^{a}\right)^{\bar{\imath}} K_{\bar{\imath} j}\left(T^{a} \phi\right)^{j}\right],
$$

where $\Lambda_{\mathrm{UV}}$ is an UV cutoff, $W_{i j}=\partial^{2} W / \partial \phi_{i} \partial \phi_{j}, K_{\bar{\imath} j}=\partial^{2} K / \partial \phi_{\bar{\imath}}^{*} \partial \phi_{j}, K^{i \bar{n}} K_{\bar{n} j}=\delta_{j}^{i}, T^{a}$ are the generators of the gauge group, and we have considered a diagonal kinetic function $f_{a b}=f_{a} \delta_{a b}$. By applying eq. (2.3) to our case, one can extract the corrections to the 
Kähler terms $H_{1}^{\dagger} H_{1}, L^{\dagger} L$ and $\left(H_{1}^{\dagger} L\right)^{2}$. The relevant RGEs are derived by combining wave function and vertex corrections. In fact, the RGE for $\boldsymbol{\kappa}$ was obtained in this way in $[37,38]$. This method allows us to derive the RGEs for $\mathbf{B}_{\kappa}, \tilde{\mathbf{B}}_{\kappa}$ and $\mathbf{C}_{\kappa}$ as well, by retaining the dependence of $K, W$ and $f_{a}$ on the spurion superfield $X=\theta^{2} F_{X}$, which effectively generates all SUSY-breaking mass parameters. We recall that gaugino masses appear in $f_{a}=\frac{1}{g_{a}^{2}}\left(1-2 \theta^{2} M_{a}\right)$, scalar masses stem from $K$ as $\left(1-\theta^{2} \bar{\theta}^{2} \tilde{m}^{2}\right) \phi^{\dagger} \phi$, while Yukawa and SUSY-breaking trilinear couplings come from $W$ through combinations like $\left(\mathbf{Y}_{e}-\theta^{2} \mathbf{A}_{e}\right) H_{1} E^{c} L$. Some of the loop-induced terms in $(\Delta K)_{\log }$ have the form $\theta^{2} \phi^{\dagger} \phi$ (or $\left.\bar{\theta}^{2} \phi^{\dagger} \phi\right)$ and can be included in a $\theta$-dependent ( $\bar{\theta}$-dependent) wave function renormalization of the superfield $\phi\left(\phi^{\dagger}\right)$ [61]. Our final result for the RGEs is:

$$
\begin{aligned}
8 \pi^{2} \frac{d \boldsymbol{\kappa}}{d t}= & {\left[g^{2}+g^{\prime 2}+\operatorname{Tr}\left(\mathbf{Y}_{e}^{\dagger} \mathbf{Y}_{e}+3 \mathbf{Y}_{d}^{\dagger} \mathbf{Y}_{d}\right)\right] \boldsymbol{\kappa}-\frac{1}{2}\left[\boldsymbol{\kappa} \mathbf{Y}_{e}^{\dagger} \mathbf{Y}_{e}+\left(\mathbf{Y}_{e}^{\dagger} \mathbf{Y}_{e}\right)^{T} \boldsymbol{\kappa}\right] } \\
8 \pi^{2} \frac{d \mathbf{B}_{\kappa}}{d t}= & {\left[g^{2}+g^{\prime 2}+\operatorname{Tr}\left(\mathbf{Y}_{e}^{\dagger} \mathbf{Y}_{e}+3 \mathbf{Y}_{d}^{\dagger} \mathbf{Y}_{d}\right)\right] \mathbf{B}_{\kappa}-\frac{1}{2}\left[\mathbf{B}_{\kappa} \mathbf{Y}_{e}^{\dagger} \mathbf{Y}_{e}+\left(\mathbf{Y}_{e}^{\dagger} \mathbf{Y}_{e}\right)^{T} \mathbf{B}_{\kappa}\right] } \\
& +\left[g^{2} M_{2}+g^{\prime 2} M_{1}\right] \boldsymbol{\kappa}, \\
8 \pi^{2} \frac{d \tilde{\mathbf{B}}_{\kappa}}{d t}= & {\left[g^{2}+g^{\prime 2}+\operatorname{Tr}\left(\mathbf{Y}_{e}^{\dagger} \mathbf{Y}_{e}+3 \mathbf{Y}_{d}^{\dagger} \mathbf{Y}_{d}\right)\right] \tilde{\mathbf{B}}_{\kappa}-\frac{1}{2}\left[\tilde{\mathbf{B}}_{\kappa} \mathbf{Y}_{e}^{\dagger} \mathbf{Y}_{e}+\left(\mathbf{Y}_{e}^{\dagger} \mathbf{Y}_{e}\right)^{T} \tilde{\mathbf{B}}_{\kappa}\right] } \\
& +\left[g^{2} M_{2}^{*}+g^{\prime 2} M_{1}^{*}-2 \operatorname{Tr}\left(\mathbf{A}_{e}^{\dagger} \mathbf{Y}_{e}+3 \mathbf{A}_{d}^{\dagger} \mathbf{Y}_{d}\right)\right] \boldsymbol{\kappa}+\boldsymbol{\kappa} \mathbf{A}_{e}^{\dagger} \mathbf{Y}_{e}+\left(\mathbf{A}_{e}^{\dagger} \mathbf{Y}_{e}\right)^{T} \boldsymbol{\kappa}, \\
8 \pi^{2} \frac{d \mathbf{C}_{\kappa}}{d t}= & {\left[g^{2}+g^{\prime 2}+\operatorname{Tr}\left(\mathbf{Y}_{e}^{\dagger} \mathbf{Y}_{e}+3 \mathbf{Y}_{d}^{\dagger} \mathbf{Y}_{d}\right)\right] \mathbf{C}_{\kappa}-\frac{1}{2}\left[\mathbf{C}_{\kappa} \mathbf{Y}_{e}^{\dagger} \mathbf{Y}_{e}+\left(\mathbf{Y}_{e}^{\dagger} \mathbf{Y}_{e}\right)^{T} \mathbf{C}_{\kappa}\right] } \\
& +\left[g^{2} M_{2}^{*}+g^{\prime 2} M_{1}^{*}-2 \operatorname{Tr}\left(\mathbf{A}_{e}^{\dagger} \mathbf{Y}_{e}+3 \mathbf{A}_{d}^{\dagger} \mathbf{Y}_{d}\right)\right] \mathbf{B}_{\kappa}+\mathbf{B}_{\kappa} \mathbf{A}_{e}^{\dagger} \mathbf{Y}_{e}+\left(\mathbf{A}_{e}^{\dagger} \mathbf{Y}_{e}\right)^{T} \mathbf{B}_{\kappa} \\
& +\left[g^{2} M_{2}+g^{\prime 2} M_{1}\right] \tilde{\mathbf{B}}_{\kappa}+4\left[2 g^{2}\left|M_{2}\right|^{2}+g^{\prime 2}\left|M_{1}\right|^{2}\right] \boldsymbol{\kappa}-\boldsymbol{\kappa} \mathbf{P}-\mathbf{P}^{T} \boldsymbol{\kappa},
\end{aligned}
$$

where $\mathbf{P} \equiv \mathbf{A}_{e}^{\dagger} \mathbf{A}_{e}+\left(\mathbf{m}_{\tilde{L}}^{2}\right)^{T} \mathbf{Y}_{e}^{\dagger} \mathbf{Y}_{e}+\mathbf{Y}_{e}^{\dagger}\left(\mathbf{m}_{\tilde{e}^{c}}^{2}\right)^{T} \mathbf{Y}_{e}+m_{H_{1}}^{2} \mathbf{Y}_{e}^{\dagger} \mathbf{Y}_{e}$. These RGEs hold in the MSSM or in its extensions with extra states that do not couple to either $H_{1}$ or $L$. The generalization to models with such extra couplings is straightforward. For instance, the NMSSM superpotential couplings $\left(\lambda_{S}-\theta^{2} A_{S}\right) S H_{2} H_{1}$ only lead to a few extra terms in the RGEs. In practice, it is enough to shift $\operatorname{Tr}\left(\mathbf{Y}_{e}^{\dagger} \mathbf{Y}_{e}\right) \rightarrow \operatorname{Tr}\left(\mathbf{Y}_{e}^{\dagger} \mathbf{Y}_{e}\right)+\left|\lambda_{S}\right|^{2}$ and $\operatorname{Tr}\left(\mathbf{A}_{e}^{\dagger} \mathbf{Y}_{e}\right) \rightarrow$ $\operatorname{Tr}\left(\mathbf{A}_{e}^{\dagger} \mathbf{Y}_{e}\right)+A_{S}^{*} \lambda_{S}$ in the above equations.

In general, eqs. (2.4)-(2.7) form a system of coupled RGEs, which exhibits operator mixing. Each equation contains a 'homogeneous' part, which is common to all four operators. Those of $\mathbf{B}_{\kappa}$ and $\tilde{\mathbf{B}}_{\kappa}$ have an additional piece which is driven by $\boldsymbol{\kappa}$ and depends on the gaugino masses and trilinear couplings. As for the RGE of $\mathbf{C}_{\kappa}$, its inhomogenous part also contains $\mathbf{B}_{\kappa}$ and $\tilde{\mathbf{B}}_{\kappa}$. Notice that the RGEs involve several independent parameters and flavour structures. Still, important simplifications may occur in specific scenarios (see sections 3 and 4 ).

For completeness, we also present the RGEs of the $d=5$ superpotential operator $\int d^{2} \theta \frac{1}{2 M_{5}}\left(\boldsymbol{\kappa}_{5}+\theta^{2} \mathbf{B}_{5}\right)_{i j}\left(L_{i} H_{2}\right)\left(L_{j} H_{2}\right)$ :

$$
8 \pi^{2} \frac{d \boldsymbol{\kappa}_{5}}{d t}=-\left[3 g^{2}+g^{\prime 2}-3 \operatorname{Tr}\left(\mathbf{Y}_{u}^{\dagger} \mathbf{Y}_{u}\right)\right] \boldsymbol{\kappa}_{5}+\frac{1}{2}\left[\boldsymbol{\kappa}_{5} \mathbf{Y}_{e}^{\dagger} \mathbf{Y}_{e}+\left(\mathbf{Y}_{e}^{\dagger} \mathbf{Y}_{e}\right)^{T} \boldsymbol{\kappa}_{5}\right]
$$



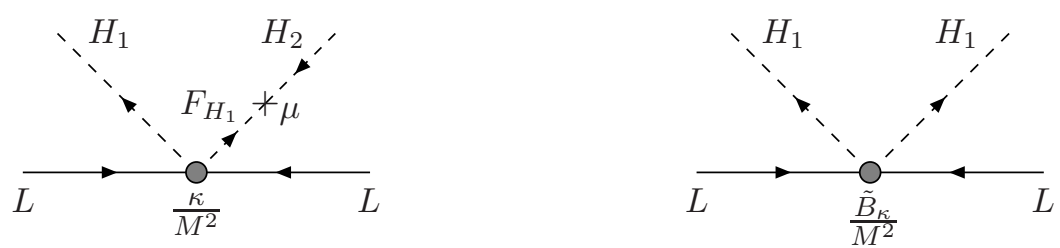

Figure 2. Tree-level contributions to neutrino masses from the Kähler operators $\left(H_{1}^{\dagger} L\right)^{2}$ and $X^{\dagger}\left(H_{1}^{\dagger} L\right)^{2}$.

$$
\begin{aligned}
8 \pi^{2} \frac{d \mathbf{B}_{5}}{d t}= & -\left[3 g^{2}+g^{\prime 2}-3 \operatorname{Tr}\left(\mathbf{Y}_{u}^{\dagger} \mathbf{Y}_{u}\right)\right] \mathbf{B}_{5}+\frac{1}{2}\left[\mathbf{B}_{5} \mathbf{Y}_{e}^{\dagger} \mathbf{Y}_{e}++\left(\mathbf{Y}_{e}^{\dagger} \mathbf{Y}_{e}\right)^{T} \mathbf{B}_{5}\right] \\
& -2\left[3 g^{2} M_{2}+g^{\prime 2} M_{1}+3 \operatorname{Tr}\left(\mathbf{Y}_{u}^{\dagger} \mathbf{A}_{u}\right)\right] \boldsymbol{\kappa}_{5}-\boldsymbol{\kappa}_{5}\left(\mathbf{Y}_{e}^{\dagger} \mathbf{A}_{e}\right)-\left(\mathbf{Y}_{e}^{\dagger} \mathbf{A}_{e}\right)^{T} \boldsymbol{\kappa}_{5}
\end{aligned}
$$

The equation for $\boldsymbol{\kappa}_{5}$ is well known $[62,63]$, while that for the corresponding SUSY-breaking parameter $\mathbf{B}_{5}$ is another novel result.

\subsection{Tree-level contributions to neutrino masses}

Consider now the effective Kähler operators of eq. (2.2), renormalized at the weak scale. Two of them, namely those with coefficients $\boldsymbol{\kappa}$ and $\tilde{\mathbf{B}}_{\kappa}$ (see figure 2), contribute directly to the neutrino mass matrix $\left(\mathcal{L} \supset-\frac{1}{2}\left(\mathbf{m}_{\nu}\right)_{i j} \nu_{i} \nu_{j}+\right.$ h.c. $)$ :

$$
\mathbf{m}_{\nu}=\mathbf{m}_{\nu}^{(\kappa)}+\mathbf{m}_{\nu}^{\left(\tilde{B}_{\kappa}\right)}
$$

The $\boldsymbol{\kappa}$-operator gives a lagrangian operator of the form $\left(F_{H_{1}}^{\dagger} L\right)\left(H_{1}^{\dagger} L\right)$, which reduces to $-\mu\left(H_{2} L\right)\left(H_{1}^{\dagger} L\right)$ after replacing $F_{H_{1}}^{\dagger} \rightarrow-\mu H_{2}$. Upon setting the Higgs fields to their VEVs $\left(\left\langle H_{1}^{0}\right\rangle=v \cos \beta,\left\langle H_{2}^{0}\right\rangle=v \sin \beta\right)$ one gets $[37,38]$

$$
\mathbf{m}_{\nu}^{(\kappa)}=2 \kappa \mu \frac{v^{2}}{M^{2}} \sin \beta \cos \beta .
$$

On the other hand, the $\tilde{\mathbf{B}}_{\kappa}$-operator leads to a lagrangian term of the form $\left(H_{1}^{\dagger} L\right)^{2}$, which induces

$$
\mathbf{m}_{\nu}^{\left(\tilde{B}_{\kappa}\right)}=\tilde{\mathbf{B}}_{\kappa} \frac{v^{2}}{M^{2}} \cos ^{2} \beta .
$$

This novel contribution to the neutrino mass matrix can be of the same order of $\mathbf{m}_{\nu}^{(\kappa)}$. In general, both the flavour structure and the relative size of $\mathbf{m}_{\nu}^{(\kappa)}$ and $\mathbf{m}_{\nu}^{\left(\tilde{B}_{\kappa}\right)}$ are model dependent. For instance, a large value of $\tan \beta$ suppresses $\mathbf{m}_{\nu}^{\left(\tilde{B}_{\kappa}\right)}$ with respect to $\mathbf{m}_{\nu}^{(\kappa)}$, whereas a hierarchy $\tilde{\mathbf{B}}_{\kappa} \gg \boldsymbol{\kappa} \mu$ enhances it (see sections 3 and 4 ).

\subsection{Radiative finite contributions to $\mathrm{m}_{\nu}$}

Additional contributions to $\mathbf{m}_{\nu}$ arise from quantum effects. Since we have already discussed the logarithmic renormalization between high and low scales, we now turn to the analysis of finite quantum corrections to $\mathbf{m}_{\nu}$ at the weak scale, i.e., at the sparticle threshold (see figure 3). In particular, we will focus on effects which exhibit potential enhancement factors, such as large values of $\tan \beta$ or large mass ratios. 

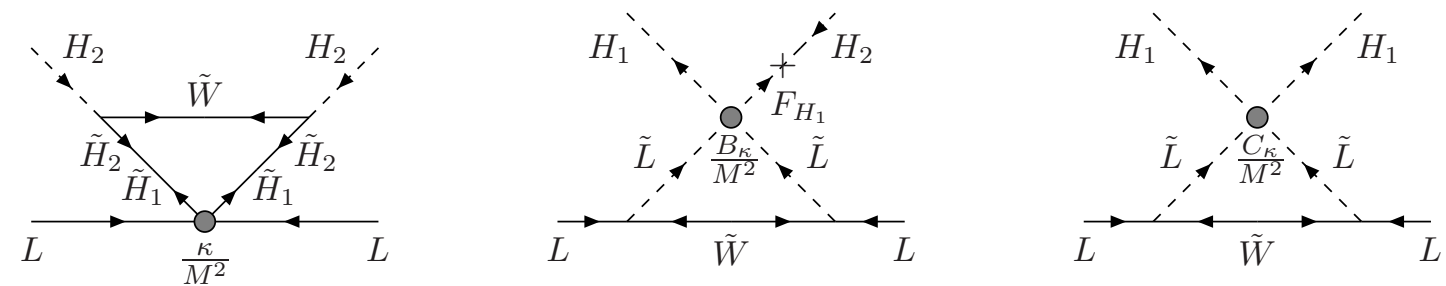

Figure 3. One-loop finite contributions to neutrino masses from the Kähler operators $\left(H_{1}^{\dagger} L\right)^{2}$, $X\left(H_{1}^{\dagger} L\right)^{2}$ and $X X^{\dagger}\left(H_{1}^{\dagger} L\right)^{2}$ (from left to right). These diagrams generate lagrangian operators of the form $\left(H_{2} L\right)^{2},\left(H_{2} L\right)\left(H_{1}^{\dagger} L\right)$ and $\left(H_{1}^{\dagger} L\right)^{2}$ (the $W$-ino can be replaced by a $B$-ino everywhere).

Consider again the $\boldsymbol{\kappa}$-operator of eq. (2.2). One of its lagrangian components is a four fermion operator with two leptons and two higgsinos, which can be dressed by a finite Higgsino-gaugino loop (left diagram in figure 3), generating an effective lagrangian term of the form $\left(\mathrm{H}_{2} L\right)^{2}$. Curiously, this reminds the 'holomorphic' structure of the familiar $d=5$ superpotential operator, but of course it cannot be interpreted that way (it arises radiatively through SUSY breaking). The resulting contribution to $\mathbf{m}_{\nu}$ is proportional to the tree-level term $\mathbf{m}_{\nu}^{(\kappa)}$ [see eq. (2.11)]:

$$
\delta_{\kappa} \mathbf{m}_{\nu}=\frac{1}{64 \pi^{2}}\left(\frac{g^{2}}{M_{2}^{*}} f_{\mu 2}+\frac{g^{\prime 2}}{M_{1}^{*}} f_{\mu 1}\right) \mu \tan \beta \mathbf{m}_{\nu}^{(\kappa)}
$$

where $f_{\mu a}=f\left(|\mu|^{2} /\left|M_{a}\right|^{2}\right)$ and $f(x)=(x-1-\log x) /(x-1)^{2}$. Despite the potential tan $\beta$ enhancement, this correction is below $2 \%$ for any ratios $\mu / M_{a}$ and any $\tan \beta<50$.

Concerning the $\mathbf{B}_{\kappa}$ and $\mathbf{C}_{\kappa}$-operators of eq. (2.2), the component expansion of the former includes a lagrangian term $\left(F_{H_{1}}^{\dagger} \tilde{L}\right)\left(H_{1}^{\dagger} \tilde{L}\right)$ which gives $-\mu\left(H_{2} \tilde{L}\right)\left(H_{1}^{\dagger} \tilde{L}\right)$, while the latter leads to $\left(H_{1}^{\dagger} \tilde{L}\right)^{2}$. Both these terms generate small $\Delta L=2$ corrections to sneutrino masses $\left(\mathcal{L} \supset-\frac{1}{2}\left(\delta \mathbf{m}_{\tilde{\nu}}^{2}\right)_{i j} \tilde{\nu}_{i} \tilde{\nu}_{j}+\right.$ h.c. $)$, namely

$$
\delta \mathbf{m}_{\tilde{\nu}}^{2}=-\left(2 \mu \mathbf{B}_{\kappa} \sin \beta \cos \beta+\mathbf{C}_{\kappa} \cos ^{2} \beta\right) \frac{v^{2}}{M^{2}},
$$

which induce tiny splittings in the sneutrino spectrum. This property of our $d=6$ operators generalizes a known effect of $d=5$ type I $[64,65]$ and type II $[40,41]$ seesaw realizations, ${ }^{3}$ and is potentially relevant for the phenomenon of sneutrino oscillations [64, 65]. Furthermore, the presence of $\Delta L=2$ scalar operators induces neutrino masses at the one-loop level, as in [64-67]. In our framework, the lagrangian terms $\left(H_{2} \tilde{L}\right)\left(H_{1}^{\dagger} \tilde{L}\right)$ and $\left(H_{1}^{\dagger} \tilde{L}\right)^{2}$ can be dressed by finite slepton-gaugino loops (middle and right diagrams in figure 3), inducing effective lagrangian operators of the form $\left(H_{2} L\right)\left(H_{1}^{\dagger} L\right)$ and $\left(H_{1}^{\dagger} L\right)^{2}$, respectively.

In order to discuss the $\mathbf{B}_{\kappa}$ and $\mathbf{C}_{\kappa}$ contributions to $\mathbf{m}_{\nu}$, we parametrize the soft mass matrix of 'left-handed' sleptons $\tilde{L}$ as $\mathbf{m}_{\tilde{L}}^{2}=\tilde{m}_{L}^{2}\left(1+\boldsymbol{\Delta}_{L}\right)$, where $\tilde{m}_{L}^{2}$ sets the overall mass

\footnotetext{
${ }^{3}$ In the $d=5$ case, the operators shown before eqs. (2.8) and (2.9) induce neutrino masses $\mathbf{m}_{\nu}=$ $\kappa_{5} \sin ^{2} \beta v^{2} / M_{5}$ as well as $\Delta L=2$ sneutrino masses $\delta \mathbf{m}_{\tilde{\nu}}^{2}=-\left(2 \mu^{*} \boldsymbol{\kappa}_{5} \sin \beta \cos \beta+\mathbf{B}_{5} \sin ^{2} \beta\right) v^{2} / M_{5}$.
} 
scale and the dimensionless matrix $\boldsymbol{\Delta}_{L}$ accounts for flavour dependence. ${ }^{4}$ At first order in $\boldsymbol{\Delta}_{L}$, the contributions to the neutrino mass matrix induced by $\mathbf{B}_{\kappa}$ and $\mathbf{C}_{\kappa}$ are:

$$
\begin{aligned}
\delta_{B_{\kappa}} \mathbf{m}_{\nu} \simeq \frac{1}{32 \pi^{2}}[- & \left(\frac{g^{2}}{M_{2}} f_{L 2}+\frac{g^{\prime 2}}{M_{1}} f_{L 1}\right) \mathbf{B}_{\kappa} \\
& \left.+\left(\frac{g^{2}}{M_{2}} h_{L 2}+\frac{g^{\prime 2}}{M_{1}} h_{L 1}\right)\left(\mathbf{B}_{\kappa} \boldsymbol{\Delta}_{L}+\boldsymbol{\Delta}_{L}^{T} \mathbf{B}_{\kappa}\right)\right] 2 \mu \frac{v^{2}}{M^{2}} \sin \beta \cos \beta \\
\delta_{C_{\kappa}} \mathbf{m}_{\nu} \simeq \frac{1}{32 \pi^{2}}[- & \left(\frac{g^{2}}{M_{2}} f_{L 2}+\frac{g^{\prime 2}}{M_{1}} f_{L 1}\right) \mathbf{C}_{\kappa} \\
& \left.+\left(\frac{g^{2}}{M_{2}} h_{L 2}+\frac{g^{\prime 2}}{M_{1}} h_{L 1}\right)\left(\mathbf{C}_{\kappa} \boldsymbol{\Delta}_{L}+\boldsymbol{\Delta}_{L}^{T} \mathbf{C}_{\kappa}\right)\right] \frac{v^{2}}{M^{2}} \cos ^{2} \beta
\end{aligned}
$$

where $f_{L a}=f\left(\tilde{m}_{L}^{2} /\left|M_{a}\right|^{2}\right), h_{L a}=h\left(\tilde{m}_{L}^{2} /\left|M_{a}\right|^{2}\right), h(x)=\left(x^{2}-1-2 x \log x\right) /(x-1)^{3}$ and $f(x)$ was defined after eq. (2.13). Both the flavour structure and the size of $\delta_{B_{\kappa}} \mathbf{m}_{\nu}, \delta_{C_{\kappa}} \mathbf{m}_{\nu}$ are model dependent. The flavour dependence enters through $\mathbf{B}_{\kappa}, \mathbf{C}_{\kappa}$ and $\boldsymbol{\Delta}_{L}$, while the overall size crucially depends on the magnitude of the SUSY-breaking parameters. Regarding the latter aspect, let us compare $\delta_{B_{\kappa}} \mathbf{m}_{\nu}$ and $\delta_{C_{\kappa}} \mathbf{m}_{\nu}$ with the tree-level terms $\mathbf{m}_{\nu}^{(\kappa)}$ and $\mathbf{m}_{\nu}^{\left(\tilde{B}_{\kappa}\right)}$ of eqs. (2.11) and (2.12). Suppose there are two SUSY-breaking mass scales $\tilde{m}$ and $\tilde{m}_{\kappa}$, such that sleptons and gauginos have masses of order $\tilde{m}$, while the SUSY-breaking terms in eq. (2.2) scale $\operatorname{as}^{5} B_{\kappa} \sim \tilde{B}_{\kappa} \sim \kappa \tilde{m}_{\kappa}, C_{\kappa} \sim \kappa \tilde{m}_{\kappa}^{2}$. Then, the relative corrections $\delta_{B_{\kappa}} m_{\nu} / m_{\nu}^{(\kappa)}$ and $\delta_{C_{\kappa}} m_{\nu} / m_{\nu}^{\left(\tilde{B}_{\kappa}\right)}$ are of order $10^{-3} \tilde{m}_{\kappa} / \tilde{m}$. In particular, they are negligible for $\tilde{m}_{\kappa} \sim \tilde{m}$ while they can be $\mathcal{O}(10 \%)$ for $\tilde{m}_{\kappa} \sim 10^{2} \tilde{m}$, as in the case of the explicit model presented in section 4 .

\section{Type II seesaw realizations}

At this point, a natural question arises about the possible origin of the $d=6, \Delta L=2$ effective operators discussed, so far, in a general way. By considering simple scenarios like the type I/II/III seesaw mechanisms (which generate the familiar $d=5$ superpotential operator at the tree level), we immediately realize that the type II framework is the natural one in which those $d=6$ operators emerge. As a matter of fact, the tree-level exchange of type I or type III mediators leads to $\Delta L=0$ Kähler operators of the form $\left|H_{2} L\right|^{2}$, whereas the type II mediators induce both $\Delta L=0$ and $\Delta L=2$ operators.

\subsection{Type II in the SUSY limit}

The type II seesaw mechanism is realized through the exchange of $\mathrm{SU}(2)_{W}$ triplet states $T=\left(T^{0}, T^{+}, T^{++}\right)$and $\bar{T}=\left(\bar{T}^{0}, \bar{T}^{-}, \bar{T}^{--}\right)$in a vector-like $\mathrm{SU}(2)_{W} \times \mathrm{U}(1)_{Y}$ representation,

\footnotetext{
${ }^{4}$ A flavour violating $\boldsymbol{\Delta}_{L}$ generically appears in models in which neutrino masses arise through coupling to heavy states. The first such examples in the type I [68] and type II [39] seesaws relied on renormalization effects. In the type II model presented in section 4 a non-vanishing $\boldsymbol{\Delta}_{L}$ is generated by finite radiative corrections at the scale of SUSY-breaking mediation.

${ }^{5}$ We replace bold characters with unbolded ones whenever we discuss order of magnitude estimates for some quantity.
} 
$T \sim(3,1), \bar{T} \sim(3,-1)$. The relevant superpotential terms are:

$$
W \supset \frac{1}{\sqrt{2}} \mathbf{Y}_{T}^{i j} L_{i} T L_{j}+\frac{1}{\sqrt{2}} \lambda_{1} H_{1} T H_{1}+\frac{1}{\sqrt{2}} \lambda_{2} H_{2} \bar{T} H_{2}+M_{T} T \bar{T},
$$

where $\mathbf{Y}_{T}^{i j}$ is a $3 \times 3$ symmetric matrix, $\lambda_{1,2}$ are dimensionless couplings and $M_{T}$ is the (SUSY) triplet mass. Neutrino masses are usually generated through the $d=5$ effective superpotential term $\frac{\lambda_{2}}{2 M_{T}} \mathbf{Y}_{T}^{i j}\left(L_{i} H_{2}\right)\left(L_{j} H_{2}\right)$, which is the leading $\Delta L=2$ operator emerging from the exchange of the triplet states. We assume this contribution to be strongly suppressed (absent) by a very small (vanishing) value of $\lambda_{2}$. This can be either imposed ad hoc or justified by symmetry arguments, like those presented in section 2.1. For instance, the smallness of $\lambda_{2}$ could be related to the smallness of $\mu$ (e.g., if we assign zero PQ charge to $\lambda_{1}$ and $M_{T}$, then we expect $\lambda_{2} \sim \mu^{2} / \Lambda_{*}^{2}$ since the PQ charge of $\lambda_{2}$ is twice that of $\mu$ ). In a NMSSM framework, simple $Z_{3}$ assignments can forbid $\lambda_{2}$ and allow the remaining terms in eq. (3.1) (e.g., one can assign $Z_{3}$ charge $-1 / 3$ to $\bar{T}$ and $1 / 3$ to all the other fields).

Once $\lambda_{2}$ is disregarded, the leading $\Delta L=2$ operator is precisely the Kähler operator $\left(H_{1}^{\dagger} L\right)^{2}$. Indeed, we can integrate out the heavy states by imposing $\partial W / \partial T=0$ and plugging the expression of $\bar{T}$ into the canonical Kähler term $\int d^{4} \theta \bar{T}^{\dagger} \bar{T}$. As a result, the following $\Delta L=2$ effective operator is generated at the scale $M_{T}$ :

$$
K_{\text {eff }} \supset \frac{\lambda_{1}^{*}}{2\left|M_{T}\right|^{2}} \mathbf{Y}_{T}^{i j}\left(H_{1}^{\dagger} L_{i}\right)\left(H_{1}^{\dagger} L_{j}\right)+\text { h.c. },
$$

along with other $\Delta L=0$ operators. The above term can be matched to the SUSY part of eq. (2.1) [or eq. (2.2)] through the identification

$$
\boldsymbol{\kappa}=\lambda_{1}^{*} \mathbf{Y}_{T} \quad, \quad M^{2}=\left|M_{T}\right|^{2} .
$$

The resulting contribution to neutrino masses is the tree-level term $\mathbf{m}_{\nu}^{(\kappa)}$ of eq. (2.11). Its diagrammatic interpretation is shown in the left diagram of figure 4, which is the explicit realization of the left diagram of figure 2. The appearance of such a contribution to neutrino masses through triplet exchange was noticed in [39] (and also in [69], where $\mu$ was generated by the VEV of a SM singlet $S$, charged under an extra U(1)). As anticipated in the Introduction, the proper neutrino masse scale is naturally reproduced by choosing $\left|M_{T}\right| \sim 10^{8}-10^{9} \mathrm{GeV}$, provided the Yukawa couplings $\lambda_{1}$ and $\mathbf{Y}_{T}$ are not suppressed. In principle the heavy triplet states could be rendered accessible to LHC by lowering $\left|M_{T}\right|$ down to the $\mathrm{TeV}$ scale. However, this rather extreme situation requires a strong suppression of those couplings.

\subsection{Type II with broken SUSY}

We now address the question on how the SUSY-breaking operators of eqs. (2.1) or (2.2) can arise in the type II seesaw framework. To this purpose, we distinguish three SUSYbreaking scenarios, depending on the ordering of the SUSY-breaking mediation scale $M_{S}$ and the triplet mass $M_{T}$ : i) $M_{S}<M_{T}$; ii) $M_{S}>M_{T}$; iii) $M_{S}=M_{T}$. 

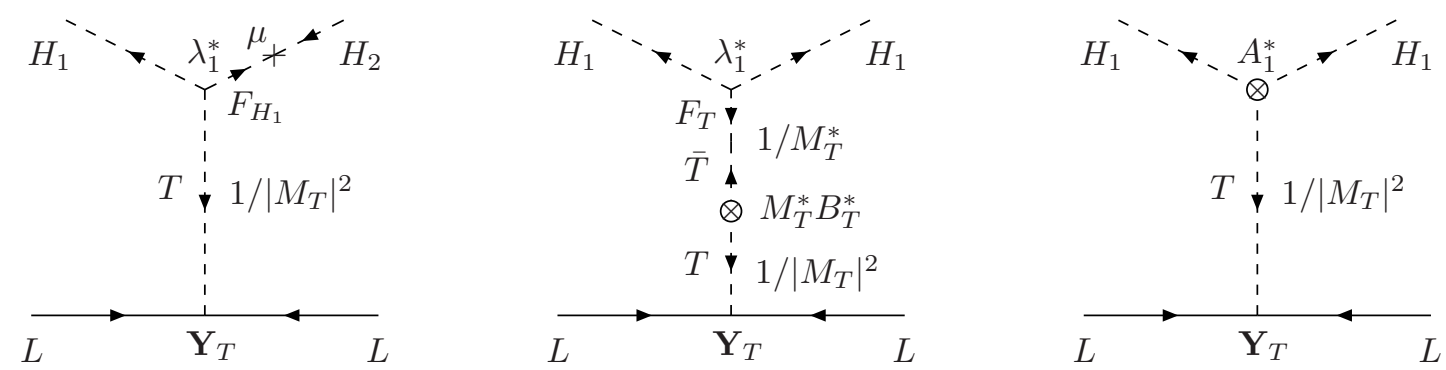

Figure 4. Tree-level contributions to neutrino masses from heavy triplet exchange.

i) $M_{S}<M_{T}$. Suppose that SUSY breaking is mediated at a low scale $M_{S}<M_{T}$ by a messenger sector coupled to the MSSM states through gauge interactions only (pure gauge mediation [70-78]). In this case, SUSY-breaking gaugino and (flavour blind) sfermion masses of order $\tilde{m}$ arise at $M_{S}$ through loop diagrams, while trilinear couplings are mainly generated below $M_{S}$ by gaugino mass terms in the RGEs. Moreover, the flavour structure of the sfermion masses and trilinear couplings emerges through RGEs only and is entirely controlled by the Yukawa matrices. Regarding our $\Delta L=2$ operators of eq. (2.2), in this scenario only the SUSY one with coefficient $\boldsymbol{\kappa}$ exists above $M_{S}$ (it is generated at $M_{T}$ ). The SUSY-breaking parameters $\mathbf{B}_{\kappa}, \tilde{\mathbf{B}}_{\kappa}$ and $\mathbf{C}_{\kappa}$ receive two-loop finite contributions at $M_{S}$, proportional to $\boldsymbol{\kappa}$, and important corrections are generated below $M_{S}$ by gaugino or scalar masses through RGEs. At low-energy, the flavour structure of such parameters is controlled by $\boldsymbol{\kappa}$ and $\mathbf{Y}_{e}$, while their expected size is $\mathbf{B}_{\kappa}, \tilde{\mathbf{B}}_{\kappa} \ll \boldsymbol{\kappa} \tilde{m}$ and $\mathbf{C}_{\kappa} \sim \boldsymbol{\kappa} \tilde{m}^{2}$. Therefore, in such a scenario the dominant source of neutrino masses is, generically, the SUSY contribution $\mathbf{m}_{\nu}^{(\kappa)}$ of eq. (2.11), while the SUSY-breaking contributions $\mathbf{m}_{\nu}^{\left(\tilde{B}_{\kappa}\right)}, \delta_{B_{\kappa}} \mathbf{m}_{\nu}$ and $\delta_{C_{\kappa}} \mathbf{m}_{\nu}$ of eqs. (2.12), (2.15) and (2.16) are subleading. We also remark that these comments do not rely on the specific realization of $\boldsymbol{\kappa}$ described in section 3.1, but hold in general for gauge-mediated SUSY breaking at scales $M_{S}<M$.

ii) $M_{S}>M_{T}$. Suppose that SUSY-breaking terms are generated at a high scale $M_{S}>$ $M_{T}$ through, e.g., gravity or gauge mediation. This implies that at $M_{T}$ all the MSSM and triplet fields generically have SUSY-breaking mass parameters. In particular, we can write those related to the triplets by replacing $M_{T} \rightarrow M_{T}\left(1-\theta^{2} B_{T}\right), \lambda_{1} \rightarrow$ $\lambda_{1}-\theta^{2} A_{1}, \mathbf{Y}_{T} \rightarrow \mathbf{Y}_{T}-\theta^{2} \mathbf{A}_{T}$ in the superpotential terms (3.1) and $\int d^{4} \theta\left[T^{\dagger} T+\right.$ $\left.\bar{T}^{\dagger} \bar{T}\right] \rightarrow \int d^{4} \theta\left[\left(1-\theta^{2} \bar{\theta}^{2} m_{T}^{2}\right) T^{\dagger} T+\left(1-\theta^{2} \bar{\theta}^{2} m_{\bar{T}}^{2}\right) \bar{T}^{\dagger} \bar{T}\right]$ in the canonical Kähler part. As previously done, we can integrate out the triplet states, including now such SUSYbreaking effects. In this way, we obtain all the $\Delta L=2$ effective operators of eq. (2.2), namely, both the SUSY one with $\boldsymbol{\kappa}=\lambda_{1}^{*} \mathbf{Y}_{T}$ [eqs. (3.2) and (3.3)] and the SUSYbreaking ones with

$$
\begin{aligned}
& \mathbf{B}_{\kappa}=\lambda_{1}^{*}\left(\mathbf{Y}_{T} B_{T}-\mathbf{A}_{T}\right), \quad \tilde{\mathbf{B}}_{\kappa}=\left(\lambda_{1}^{*} B_{T}^{*}-A_{1}^{*}\right) \mathbf{Y}_{T}, \\
& \mathbf{C}_{\kappa}=\left(\lambda_{1}^{*} B_{T}^{*}-A_{1}^{*}\right)\left(\mathbf{Y}_{T} B_{T}-\mathbf{A}_{T}\right)-\lambda_{1}^{*} \mathbf{Y}_{T} m_{\bar{T}}^{2} .
\end{aligned}
$$

We recall that $\tilde{\mathbf{B}}_{\kappa}$ contributes to neutrino masses at the tree-level $\left[\right.$ see $\mathbf{m}_{\nu}^{\left(\tilde{B}_{\kappa}\right)}$ in 
eq. (2.12)]. Its diagrammatic origin from triplet exchange is shown in the middle and right diagrams of figure 4 , which are the explicit realization of the right diagram of figure 2. The diagrammatic interpretation of $\mathbf{B}_{\kappa}$ and $\mathbf{C}_{\kappa}$ is straightforward. The relative size of the parameters which contribute to neutrino masses is model dependent, while their flavour structure exhibit remarkable features, as a consequence of the type II seesaw mechanism. In particular, $\boldsymbol{\kappa}$ and $\tilde{\mathbf{B}}_{\kappa}$ (which generate the leading contributions to neutrino masses) are aligned in flavour space at $M_{T}$, as both are proportional to $\mathbf{Y}_{T}$. Some misalignment is induced by $\mathbf{Y}_{e}$ and $\mathbf{A}_{e}$ through RGEs [see eqs. (2.4) and (2.6)]. On the other hand, $\mathbf{B}_{\kappa}$ and $\mathbf{C}_{\kappa}$ (which contribute to $\mathbf{m}_{\nu}$ through low-energy threshold corrections) owe their flavour dependence to $\mathbf{Y}_{T}$ and $\mathbf{A}_{T}$ already at $M_{T}$, and acquire further structure via RGEs. Nevertheless, if the mechanism of SUSY-breaking mediation at $M_{S}$ is flavour blind, like in minimal gravityor gauge-mediated models, all flavour structures are controlled by $\mathbf{Y}_{T}$ and $\mathbf{Y}_{e}$.

iii) $M_{S}=M_{T}$. This case deserves special attention, and we will discuss it thoroughly in the next section.

\section{Seesaw mediators as SUSY-breaking messengers}

We have seen that the type II scenario provides a natural framework to induce neutrino masses through Kähler operators. So far, the mechanisms that mediate SUSY breaking and lepton number violation have been kept distinct. Now we discuss an appealing scenario in which such mechanisms are unified, namely, the seesaw mediators are identified with the SUSY-breaking ones. This idea was proposed and thoroughly explored by two of us in $[40,41]$, where neutrino masses were generated through an effective $d=5$ superpotential in a type II scenario. Further developments were presented in [79-82] with either type II or type III mediators. We now aim at extending such an approach to our framework with $d=6$ Kähler operators. We present a minimal scenario in which a single SUSYbreaking source determines the sparticle spectrum and plays a major rôle in generating neutrino masses. Moreover, the magnitude and flavour structure of all mass parameters are closely correlated.

\subsection{SUSY-breaking mediation}

We start by identifying the type II triplets with SUSY-breaking mediators and embed them in a minimal messenger sector which, in order to generate the gluino mass, should also include coloured fields. In addition, we require that perturbative unification of gauge couplings be preserved and that all messenger masses be of the same order. This implies that the messenger sector should have the same total Dynkin index $N$ for each subgroup of $\mathrm{SU}(3)_{C} \times \mathrm{SU}(2)_{W} \times \mathrm{U}(1)_{Y}$. One way to realize this is to embed the $\mathrm{SU}(2)_{W}$ triplets $T$ and $\bar{T}$ into complete $\mathrm{SU}(5)$ representations, ${ }^{6}$ like in standard gauge mediation. The simplest such embedding is $T \subset 15$ and $\bar{T} \subset \overline{15}$, which has $N=7$ [40, 41]. Alternatively, the

\footnotetext{
${ }^{6} \mathrm{SU}(5)$ extensions of the type II seesaw with gravity-mediated SUSY breaking have been discussed in $[39,83-86]$.
} 
messenger sector could have the same $N$ for each group factor even without filling unified multiplets [87, 88]. Since $T$ and $\bar{T}$ have $\mathrm{SU}(2)$ index $N_{2}=4$, we are constrained to $N \geq 4$. In particular, we can look for a minimal messenger sector with $N=4$. One possible choice relies on adding a pair of $\mathrm{SU}(3)_{C}$ triplets $(3,1,-1 / 3)+(\overline{3}, 1,+1 / 3)$ and an $\mathrm{SU}(3)_{C}$ adjoint $(8,1,0)$ to the $T+\bar{T}$ pair. ${ }^{7}$ The octet can also be replaced by three pairs of coloured triplets, i.e. $(8,1,0) \rightarrow 3 \times[(3,1,0)+(\overline{3}, 1,0)]$. These are the only two possibilities with $N=4$ and no exotic charges.

Following the standard parametrization of minimal gauge mediation [70-78], we write messenger mass terms as $W \supset \xi_{i} X \Phi_{i} \bar{\Phi}_{i}$ (or $\xi_{i} X \Phi_{i}^{2} / 2$ for real representations), where $\left\langle\left. X\right|_{0}\right\rangle=v_{X}$ and $\left\langle\left. X\right|_{\theta^{2}}\right\rangle=F_{X}$. Thus, the messengers have SUSY masses $M_{i}=\xi_{i} v_{X}$ and a common $B$-parameter $\Lambda=F_{X} / v_{X}$, which is usually named effective SUSY-breaking scale. Hereafter, we set $\Lambda \equiv-B_{T}$ (consistently with our notation) and assume that all $\xi_{i}$ are of the same order, so that we can deal with a common messenger scale $M_{i} \sim M_{T}$.

The MSSM SUSY-breaking parameters are generated at the quantum level by a messenger sector of the type described above, coupled to the MSSM fields through both gauge and Yukawa interactions. ${ }^{8}$ At the one-loop level, the gaugino masses $M_{a}$, the Higgs $B$-term $B_{H}$ and the trilinear terms $\mathbf{A}_{x}$ are:

$$
\begin{aligned}
& M_{a}=-\frac{N B_{T}}{16 \pi^{2}} g_{a}^{2}, \quad B_{H}=\frac{3 B_{T}}{16 \pi^{2}}\left|\lambda_{1}\right|^{2}, \\
& \mathbf{A}_{e}=\frac{3 B_{T}}{16 \pi^{2}} \mathbf{Y}_{e}\left(\mathbf{Y}_{T}^{\dagger} \mathbf{Y}_{T}+\left|\lambda_{1}\right|^{2}\right), \quad \mathbf{A}_{d}=\frac{3 B_{T}}{16 \pi^{2}} \mathbf{Y}_{d}\left|\lambda_{1}\right|^{2}, \quad \mathbf{A}_{u}=0,
\end{aligned}
$$

where $g_{1}^{2}=(5 / 3) g^{\prime 2}$ and $g_{2}^{2}=g^{2}$. The result for $B_{H}$ assumes that the effective $\mu$ parameter has been generated at a scale $\Lambda_{*}>M_{T}$. We do not address this issue in detail and pragmatically suppose that the mechanism which generates $\mu$ gives vanishing or negligible contributions to the Higgs SUSY-breaking masses. Non-vanishing $\mathcal{O}\left(B_{T}^{2}\right)$ contributions for the squared scalar masses arise at the two-loop level:

$$
\begin{aligned}
\mathbf{m}_{\tilde{L}}^{2}= & \left(\frac{\left|B_{T}\right|}{16 \pi^{2}}\right)^{2}\left[N\left(\frac{3}{10} g_{1}^{4}+\frac{3}{2} g_{2}^{4}\right)-\left(\frac{27}{5} g_{1}^{2}+21 g_{2}^{2}\right) \mathbf{Y}_{T}^{\dagger} \mathbf{Y}_{T}+3\left|\lambda_{1}\right|^{2}\left(\mathbf{Y}_{T}^{\dagger} \mathbf{Y}_{T}-\mathbf{Y}_{e}^{\dagger} \mathbf{Y}_{e}\right)\right. \\
& \left.+3 \mathbf{Y}_{T}^{\dagger}\left(\mathbf{Y}_{e}^{\dagger} \mathbf{Y}_{e}\right)^{T} \mathbf{Y}_{T}+18\left(\mathbf{Y}_{T}^{\dagger} \mathbf{Y}_{T}\right)^{2}+3 \mathbf{Y}_{T}^{\dagger} \mathbf{Y}_{T} \operatorname{Tr}\left(\mathbf{Y}_{T}^{\dagger} \mathbf{Y}_{T}\right)\right] \\
\mathbf{m}_{\tilde{e}^{c}}^{2}= & \left(\frac{\left|B_{T}\right|}{16 \pi^{2}}\right)^{2}\left[N\left(\frac{6}{5} g_{1}^{4}\right)-6 \mathbf{Y}_{e}\left(\mathbf{Y}_{T}^{\dagger} \mathbf{Y}_{T}+\left|\lambda_{1}\right|^{2}\right) \mathbf{Y}_{e}^{\dagger}\right] \\
\mathbf{m}_{\tilde{Q}}^{2}= & \left(\frac{\left|B_{T}\right|}{16 \pi^{2}}\right)^{2}\left[N\left(\frac{1}{30} g_{1}^{4}+\frac{3}{2} g_{2}^{4}+\frac{8}{3} g_{3}^{4}\right)-3\left|\lambda_{1}\right|^{2} \mathbf{Y}_{d}^{\dagger} \mathbf{Y}_{d}\right] \\
\mathbf{m}_{\tilde{u}^{c}}^{2}= & \left(\frac{\left|B_{T}\right|}{16 \pi^{2}}\right)^{2}\left[N\left(\frac{8}{15} g_{1}^{4}+\frac{8}{3} g_{3}^{4}\right)\right] \\
\mathbf{m}_{\tilde{d}^{c}}^{2}= & \left(\frac{\left|B_{T}\right|}{16 \pi^{2}}\right)^{2}\left[N\left(\frac{2}{15} g_{1}^{4}+\frac{8}{3} g_{3}^{4}\right)-6\left|\lambda_{1}\right|^{2} \mathbf{Y}_{d} \mathbf{Y}_{d}^{\dagger}\right]
\end{aligned}
$$

\footnotetext{
${ }^{7}$ This is one of the 'magic' combinations listed in [88].

${ }^{8}$ In our case, the relevant Yukawa couplings are $\lambda_{1}$ and $\mathbf{Y}_{T}$. We neglect the effect of other Yukawa couplings which may involve messenger fields (see e.g. [40, 41]).
} 


$$
\begin{aligned}
m_{H_{2}}^{2}=\left(\frac{\left|B_{T}\right|}{16 \pi^{2}}\right)^{2}\left[N\left(\frac{3}{10} g_{1}^{4}+\frac{3}{2} g_{2}^{4}\right)\right] \\
m_{H_{1}}^{2}=\left(\frac{\left|B_{T}\right|}{16 \pi^{2}}\right)^{2}\left[N\left(\frac{3}{10} g_{1}^{4}+\frac{3}{2} g_{2}^{4}\right)-\left(\frac{27}{5} g_{1}^{2}+21 g_{2}^{2}\right)\left|\lambda_{1}\right|^{2}+21\left|\lambda_{1}\right|^{4}\right. \\
\left.+3\left|\lambda_{1}\right|^{2} \operatorname{Tr}\left(\mathbf{Y}_{T}^{\dagger} \mathbf{Y}_{T}+\mathbf{Y}_{e}^{\dagger} \mathbf{Y}_{e}+3 \mathbf{Y}_{d}^{\dagger} \mathbf{Y}_{d}\right)-3 \operatorname{Tr}\left(\mathbf{Y}_{T}^{\dagger} \mathbf{Y}_{T} \mathbf{Y}_{e}^{\dagger} \mathbf{Y}_{e}\right)\right]
\end{aligned}
$$

The above results follow from simple changes (including a correction of the $M_{a}$ sign) in the formulae of $[40,41]$, which were derived by applying the method $^{9}$ of [92]. Eqs. (4.1)-(4.8) form the complete set of boundary conditions at $M_{T}$ for the SUSY-breaking parameters, which must be subsequently renormalized down to low energies. Notice that the flavour structures of $\mathbf{A}_{e}, \mathbf{m}_{\tilde{L}}^{2}$ and $\mathbf{m}_{\tilde{e}^{c}}^{2}$ are controlled by $\mathbf{Y}_{T}$ and $\mathbf{Y}_{e}$, which in turn are closely related to the low-energy lepton masses and mixing angles. Such minimal LFV properties are a characteristic feature of the SUSY type II seesaw [39-41]. Clearly, our scenario possesses the property of minimal flavour violation [93-96] in both the quark and lepton sectors. The former is controlled by the usual spurions $\mathbf{Y}_{u}$ and $\mathbf{Y}_{d}$, while in the latter the matrices $\mathbf{Y}_{T}$ and $\mathbf{Y}_{e}$ are the spurions of the (minimal) lepton flavour symmetry $\mathrm{SU}(3)_{L} \times \mathrm{SU}(3)_{E^{c}}$, under which $\mathbf{Y}_{T} \sim(\overline{6}, 1)$ and $\mathbf{Y}_{e} \sim(\overline{3}, \overline{3})$. All leptonic quantities depend on invariant combinations of such spurions. For instance, the aforementioned symmetry allows $\mathbf{m}_{\tilde{L}}^{2}$ to contain structures like $\mathbf{Y}_{T}^{\dagger} \mathbf{Y}_{T}$ and $\mathbf{Y}_{e}^{\dagger} \mathbf{Y}_{e}$ at the quadratic level and $\left(\mathbf{Y}_{T}^{\dagger} \mathbf{Y}_{T}\right)^{2}, \mathbf{Y}_{T}^{\dagger}\left(\mathbf{Y}_{e}^{\dagger} \mathbf{Y}_{e}\right)^{T} \mathbf{Y}_{T},\left(\mathbf{Y}_{T}^{\dagger} \mathbf{Y}_{T}\right)\left(\mathbf{Y}_{e}^{\dagger} \mathbf{Y}_{e}\right),\left(\mathbf{Y}_{e}^{\dagger} \mathbf{Y}_{e}\right)\left(\mathbf{Y}_{T}^{\dagger} \mathbf{Y}_{T}\right),\left(\mathbf{Y}_{e}^{\dagger} \mathbf{Y}_{e}\right)^{2}$ at the quartic one. In fact, all such combinations are present in $\mathbf{m}_{\tilde{L}}^{2}$ at low energy, since some appear at $M_{T}$ [eq. (4.3)] and others are induced through RGEs. Finally, we remark that there is a phase alignment among $M_{a}, B_{H}$ and $\mathbf{A}_{x}$, induced by the common factor $B_{T}$. As a consequence, the one-loop sfermion/gaugino/higgsino contributions to the electric-dipole moments are strongly suppressed.

\subsection{Neutrino masses}

Upon decoupling the triplets, the MSSM SUSY-breaking masses are generated through finite radiative effects [eqs. (4.1)-(4.8)], while the $\Delta L=2$ SUSY-breaking parameters $\mathbf{B}_{\kappa}$, $\tilde{\mathbf{B}}_{\kappa}$ and $\mathbf{C}_{\kappa}$ arise at the tree level. The latter have a very simple form, namely that of eqs. (3.4) and (3.5) with vanishing $A_{1}, \mathbf{A}_{T}$ and $m_{\bar{T}}^{2}$ :

$$
\mathbf{B}_{\kappa}=B_{T} \boldsymbol{\kappa}, \quad \tilde{\mathbf{B}}_{\kappa}=B_{T}^{*} \boldsymbol{\kappa}, \quad \mathbf{C}_{\kappa}=\left|B_{T}\right|^{2} \boldsymbol{\kappa},
$$

where $\boldsymbol{\kappa}=\lambda_{1}^{*} \mathbf{Y}_{T}$ [eq. (3.3)]. These alignment relations hold at the scale $M_{T}$, and remain also valid to a very good approximation after $R G$ evolution, which is dominated by the homogeneous terms. Indeed, the non-homogeneous terms in the RGEs (2.5)-(2.7) are

\footnotetext{
${ }^{9}$ This method provides the leading terms of a power expansion in $\left|B_{T} / M_{T}\right|^{2}$, which we assume to be $\ll 1$. The latter condition also allows us to neglect: $i)$ corrections such as $\delta \mathbf{m}_{\tilde{L}}^{2} \simeq-\mathbf{Y}_{T}^{\dagger} \mathbf{Y}_{T}\left|B_{T}\right|^{4} /\left(32 \pi^{2}\left|M_{T}\right|^{2}\right)$ and $\delta m_{H_{1}}^{2} \simeq-\left|\lambda_{1}\right|^{2}\left|B_{T}\right|^{4} /\left(32 \pi^{2}\left|M_{T}\right|^{2}\right)$, which are the leading one-loop contributions to scalar masses; ii) quartic terms like $-\left|\lambda_{1} B_{T} / M_{T}\right|^{2}\left|H_{1}\right|^{4} / 2$, induced in the scalar potential by the tree-level exchange of the triplets; iii) four-lepton operators generated by the tree-level exchange of the triplets, which can contribute to LFV processes such as $\mu \rightarrow 3 e$ (see, e.g., [89-91]).
} 
proportional to MSSM SUSY-breaking parameters, which are loop-suppressed with respect to $B_{T}$. So, we can apply eq. (4.9) also at low scales, as long as we take into account the RG running of $\boldsymbol{\kappa}$ [eq. (2.4)]. The relative size of $\mathbf{B}_{\kappa}, \tilde{\mathbf{B}}_{\kappa}$ and $\mathbf{C}_{\kappa}$ is also completely determined and, therefore, it is simple to evaluate and compare the corresponding contributions to neutrino masses.

Inserting now the expression of $\tilde{\mathbf{B}}_{\kappa}$ from eq. (4.9) into the general eqs. (2.10)-(2.12), we can write the tree-level contribution to the neutrino mass matrix as:

$$
\mathbf{m}_{\nu}=\boldsymbol{\kappa}\left(B_{T}^{*}+2 \mu \tan \beta\right) \cos ^{2} \beta \frac{v^{2}}{\left|M_{T}\right|^{2}} .
$$

Although we have not specified the mechanism which generates $\mu$, we note that $\mu$ is suppressed with respect to $B_{T}$ since the conditions of electroweak symmetry breaking (EWSB) connect $\mu$ with other SUSY-breaking parameters, whose size $\tilde{m}$ is related to $B_{T}$ by a loop factor. Hence, we expect $\tilde{\mathbf{B}}_{\kappa}=B_{T}^{*} \boldsymbol{\kappa}$ to be the dominant source of neutrino masses in the present scenario. The contribution proportional to $\mu$ may become comparable to such leading term only for large values of $\tan \beta$.

Regarding the finite quantum corrections to $\mathbf{m}_{\nu}$ discussed in section 2.5, we conclude that $\delta_{\kappa} \mathbf{m}_{\nu}$ is very small [see eq. (2.13) and related comments]. The other contribution $\delta \mathbf{m}_{\nu}=\delta_{B_{\kappa}} \mathbf{m}_{\nu}+\delta_{C_{\kappa}} \mathbf{m}_{\nu}$ induced by $\mathbf{B}_{\kappa}$ and $\mathbf{C}_{\kappa}$ is more interesting. From eqs. (2.15) and (2.16) we get:

$$
\delta \mathbf{m}_{\nu} \simeq \frac{1}{2 N}\left(f_{L 2}+\frac{3}{5} f_{L 1}\right) \mathbf{m}_{\nu}-\frac{1}{2 N}\left(h_{L 2}+\frac{3}{5} h_{L 1}\right)\left(\mathbf{m}_{\nu} \boldsymbol{\Delta}_{L}+\boldsymbol{\Delta}_{L}^{T} \mathbf{m}_{\nu}\right)
$$

which is directly related to the tree-level term $\mathbf{m}_{\nu}$ of eq. (4.10). We also note that the parametric loop-suppression of $\delta \mathbf{m}_{\nu}$ has disappeared, since the low-energy loop factor in eqs. (2.15) and (2.16) has been compensated by the inverse loop factor in $B_{T} / M_{a}$ [see eq. (4.1)]. There is still a residual numerical suppression, which depends on the messenger index $N$ and on the low-energy values of $\tilde{m}_{L}^{2} /\left|M_{a}\right|^{2}$ entering $f_{L a}$ and $h_{L a}$. For instance, the leading correction (which is always aligned with $\left.\mathbf{m}_{\nu}\right)$ is $\delta \mathbf{m}_{\nu} \simeq 0.1 \mathbf{m}_{\nu}\left(0.07 \mathbf{m}_{\nu}\right)$ for $N=4$ $(N=7)$. Furthermore, even the small flavour-dependent term induced by $\boldsymbol{\Delta}_{L}$ does not exhibit an independent structure, since both $\boldsymbol{\Delta}_{L}$ and $\mathbf{m}_{\nu}$ are controlled by the basic lepton flavour spurions $\mathbf{Y}_{T}$ and $\mathbf{Y}_{e}$. This confirms the minimal LVF properties of the present scenario. We also recall that, in general, $\boldsymbol{\Delta}_{L}$ also induces a misalignment between $\mathbf{Y}_{e}$ and the charged lepton mass matrix $\mathcal{M}_{e}$ through $\tan \beta$-enhanced threshold corrections [97-100]. In our case, the resulting effects on the lepton mixing matrix $\mathbf{U}$ are again controlled by our minimal LFV structure and are numerically small.

Finally, it is worth mentioning that the $\Delta L=2$ sneutrino mass matrix of eq. (2.14) is directly linked to $\mathbf{m}_{\nu}$ through $\delta \mathbf{m}_{\tilde{\nu}}^{2}=B_{T} \mathbf{m}_{\nu}$, which is the same relation found in the $d=5$ realization of $[40,41]$. However, in both cases the conditions for the observability of sneutrino-antisneutrino oscillations $[64,65]$ are not fulfilled (despite the large enhancement factor $\left.B_{T} / \tilde{m}_{L}\right)$ since the oscillation frequency $\Delta m_{\tilde{\nu}} \sim \delta \mathbf{m}_{\tilde{\nu}}^{2} / \tilde{m}_{L} \sim\left(B_{T} / \tilde{m}_{L}\right) m_{\nu} \sim 10^{2} m_{\nu}$ is much smaller than the sneutrino decay width. Indeed, since two body decay channels like $\tilde{\nu} \rightarrow \nu \tilde{\chi}_{1}^{0}$ are open, we have $\Gamma_{\tilde{\nu}} \sim 10^{-3} \tilde{m}_{L}$, implying $\Delta m_{\tilde{\nu}} / \Gamma_{\tilde{\nu}} \lesssim 10^{-7}$. 


\subsection{Phenomenological viability, MSSM spectrum and LHC searches}

The above scenario has a small number of free parameters, namely $M_{T}, B_{T}, \lambda_{1}$ and the messenger index $N$. Once these are fixed, ${ }^{10}$ the remaining parameters $\mathbf{Y}_{T}, \tan \beta$ and $\mu$ are determined by the low-energy neutrino data and by requiring proper EWSB. Concerning neutrino data, we recall that the neutrino mass matrix $\mathbf{m}_{\nu}$ is related to the low-energy observables as $\mathbf{m}_{\nu}=\mathbf{U}^{*} \mathbf{m}_{\nu}^{D} \mathbf{U}^{\dagger}$, where $\mathbf{m}_{\nu}^{D}=\operatorname{diag}\left(m_{1}, m_{2}, m_{3}\right), m_{a}$ are the neutrino masses and $\mathbf{U}$ is the lepton mixing matrix. ${ }^{11}$ Several other observables are predicted, such as sparticle and Higgs masses, as well as LFV decay rates.

Before presenting a numerical analysis, we can already infer some information about the allowed parameter space by considering the parametric dependence of the neutrino mass in eq. (4.10), $m_{\nu} \sim Y_{T} \lambda_{1} \cos ^{2} \beta B_{T} v^{2} / M_{T}^{2}$. For $B_{T} \lesssim 10^{5} \mathrm{GeV}$ (which leads to a superpartner spectrum below a few $\mathrm{TeV}$, within the reach of the $\mathrm{LHC}$ ) and $Y_{T}, \lambda_{1} \lesssim 1$, a neutrino mass scale $m_{\nu} \sim 0.1 \mathrm{eV}$ requires $M_{T} \lesssim 10^{9} \mathrm{GeV}$. This upper bound on $M_{T}$ implies a non-trivial constraint on the messenger sector. Indeed, suppose we choose the simplest grand unified embedding with $T \subset 15$ and $\bar{T} \subset \overline{15}(N=7)$. In this case, the lower bound on $M_{T}$ compatible with one-loop gauge coupling unification is $M_{T} \gtrsim 10^{7} \mathrm{GeV}$. At the two-loop level, we find the stronger constraint $M_{T} \gtrsim 5 \times 10^{8} \mathrm{GeV}$. We have explored the parameter space for this scenario, taking into account the bounds on the lightest Higgs mass $m_{h}$ [101] and on rare LFV decays [102-104]. The outcome is a certain tension between these constraints and that on perturbative gauge coupling unification. In other words, a messenger sector with heavy states in $15+\overline{15}$, which is perfectly compatible with the $d=5$ realization of neutrino masses $[40,41]$, is only marginally compatible with the $d=6$ scenario proposed here. Hence, we will present quantitative results for a smaller messenger sector, namely, a minimal one with $N=4$. As mentioned in section 4.1 , this can be realized, e.g., by adding $\mathrm{SU}(3)_{C}$ triplets $(3,1,-1 / 3)+(\overline{3}, 1,+1 / 3)$ and an adjoint $(8,1,0)$ to the $T+\bar{T}$ pair. Perturbative gauge coupling unification is no longer a problem in this case, since it can be achieved with messenger masses as low as $10^{5} \mathrm{GeV}$.

Some representative numerical results for the $N=4$ scenario are shown in figure 5 . We set the scale of sparticle masses by fixing $B_{T}=60 \mathrm{TeV}$, and consider a normally ordered neutrino spectrum, with $0=m_{1}<m_{2} \ll m_{3}$ and $s_{13}=0$. In the upper part of figure 5 we show two plots of the $\left(\lambda_{1}, M_{T}\right)$ parameter space, including contours of $\tan \beta$ and $\mu$ (extracted by imposing EWSB). The left (right) panel corresponds to solutions of the EWSB conditions with $\mu M_{a}>0\left(\mu M_{a}<0\right)$. The main phenomenological constraints come from the LFV decay $\mu \rightarrow e \gamma$ and the lightest Higgs mass. ${ }^{12}$ In both panels, the upper region is excluded by large values of $\mathbf{Y}_{T}$, which either exceed the perturbative limits or generate excessive $\mathrm{LFV}$ in $\mathbf{m}_{\tilde{L}}^{2}$, so that the bound $\mathrm{BR}(\mu \rightarrow e \gamma)<1.2 \times 10^{-11}$ is violated. The plots also indicate other benchmark values of this $\mathrm{BR}$, which will be experimentally probed in the near future [106]. Other regions are excluded by the EWSB

\footnotetext{
${ }^{10}$ Hereafter the parameters $M_{T}, B_{T}$ and $\lambda_{1}$ are taken as real, without loss of generality.

${ }^{11}$ We use the standard parametrization $\mathbf{U}=\mathbf{V}\left(\theta_{12}, \theta_{23}, \theta_{13}, \delta\right) \cdot \operatorname{diag}\left(1, e^{i \phi_{1}}, e^{i \phi_{2}}\right)$. In our numerical analysis we will use the best-fit values for the neutrino parameters $\Delta m_{21}^{2}=\left(m_{2}^{2}-m_{1}^{2}\right)=7.65 \times 10^{-5} \mathrm{eV}^{2}$, $\left|\Delta m_{31}^{2}\right|=\left|m_{3}^{2}-m_{1}^{2}\right|=2.4 \times 10^{-3} \mathrm{eV}^{2}, \sin ^{2} \theta_{12}=0.3, \sin ^{2} \theta_{23}=0.5$ and the upper bound $s_{13}=\sin \theta_{13}<$ $0.2[1-13]$.

${ }^{12}$ We have included low-energy corrections to $m_{h}$ by linking our code to FeynHiggs [105].
} 
requirement. Notice that large values of $\tan \beta$ are achieved for $\mu M_{a}>0$, which entails that in the corresponding parameter space the two tree-level contributions to the neutrino mass matrix (4.10) have comparable size and opposite sign. An area in the right part of the $\mu M_{a}<0$ panel is excluded as well, since there the tree-level contribution to $m_{h}$ is suppressed by low values of $\tan \beta$, such that $m_{h}<114 \mathrm{GeV}$. Inside the allowed portions of parameter space (shown in white), $m_{h}$ is around $115 \mathrm{GeV}$. We have also shown some contours of the SUSY contribution $\delta a_{\mu}$ to the muon anomalous magnetic moment, which can have either sign in our model. ${ }^{13}$ For $\mu M_{a}>0$, the size of $\delta a_{\mu}$ and its positive sign are such that theory and experiment agree within $1 \sigma$. For $\mu M_{a}<0$ we have $\delta a_{\mu}<0$, hence the discrepancy is not better than in the SM. In this case we conservatively tolerate values of $\delta a_{\mu}$ up to $10^{-9}$ in magnitude. In the lower panel of figure 5 we show the sparticle and Higgs spectrum for $M_{T}=8 \times 10^{7} \mathrm{GeV}$ and $\lambda_{1}=0.25$ (which corresponds to $\tan \beta \simeq 11$ ), again for $B_{T}=60 \mathrm{TeV}$. The BRs of the LFV radiative decays are indicated in the caption. The Higgs sector is close to the decoupling limit, since the states $A, H$ and $H^{+}$are much heavier than $h$. Gluino and squarks are the heaviest sparticles and the lightest of them is $\tilde{t}_{1}$ (which is mainly $\tilde{t}_{R}$ ). In the electroweak sector, the heaviest chargino and neutralinos $\left(\tilde{\chi}_{2}^{+}, \tilde{\chi}_{3,4}^{0}\right)$ are mainly Higgsino-like, while $\tilde{\chi}_{1}^{+}$and $\tilde{\chi}_{2}^{0}$ are mostly Wino-like. The (mainly left-handed) sleptons $\tilde{\ell}_{4,5,6}$ and the sneutrinos $\tilde{\nu}_{1,2,3}$ are somewhat lighter than those states, and the Bino-like neutralino $\tilde{\chi}_{1}^{0}$ is even lighter. Finally, the lightest MSSM sparticles are the (mainly right-handed) sleptons $\tilde{\ell}_{1,2,3}$, as generically occurs in gauge mediated models with messenger index $N>1$ and not too large mediation scale [109-113]. The slepton $\tilde{\ell}_{1}$ (which is mainly $\tilde{\tau}_{R}$ ) is the next-to-lightest SUSY particle (NLSP), while the gravitino $\tilde{G}$ is the lightest SUSY particle (LSP). We recall that the latter has mass $m_{\tilde{G}}=F /\left(\sqrt{3} M_{P}\right)$, where $M_{P}$ is the Planck mass and $\sqrt{F}$ is the fundamental scale of SUSY breaking. ${ }^{14}$

The qualitative picture described above does not change very much under variations of the model parameters. For instance, if we increase $B_{T}$ the spectrum exhibits a roughly linear increase. The main exception is $m_{h}$, which could increase by a few $\mathrm{GeV}$, as a result of the logarithmic corrections induced by larger stop masses. Consequently, the rightmost boundary of the allowed parameter space (upper-right panel of figure 5) would be shifted towards larger (smaller) values of $\lambda_{1}(\tan \beta)$. At the same time, the upper boundaries determined by the $\mu \rightarrow e \gamma$ constraint would slightly shift upwards, since a heavier spectrum would imply smaller values for the LFV BRs. Increasing $B_{T}$ also reduces the magnitude of $\delta a_{\mu}\left(\left|\delta a_{\mu}\right| \propto 1 / B_{T}^{2}\right)$. Variations of $M_{T}$ induce logarithmic effects on the sparticle spectrum. For low values of $M_{T}$, Higgsino and Wino masses are closer to each other, and mixing effects in the chargino and neutralino sectors are more important. Moreover, the heavy Higgses

\footnotetext{
${ }^{13}$ We recall that the discrepancy $\Delta a_{\mu}=a_{\mu}^{S M}-a_{\mu}^{\exp }$ between the SM prediction and experiment has still some uncertainty, mainly related to the evaluation of the hadronic contribution to $a_{\mu}^{S M}$. For instance, refs. $[107,108]$ estimate $\Delta a_{\mu} \simeq-(2.5 \pm 0.8) \times 10^{-9}$ using $e^{+} e^{-}$data or $\Delta a_{\mu} \simeq-(1.6 \pm 0.8) \times 10^{-9}$ using $\tau$ data.

${ }^{14}$ Following the standard approach of gauge mediation models, we regard $F$ as an independent parameter, which fulfils the inequalities $F \gtrsim\left|F_{X}\right| \gtrsim\left|\xi_{T} F_{X}\right|=\left|B_{T} M_{T}\right|$. In principle $\sqrt{F}$ can vary in the range $10^{5}-$ $10^{9} \mathrm{GeV}$, which corresponds to a wide range of gravitino masses, from the eV to the $\mathrm{GeV}$ scale. These ranges could be further constrained by astrophysical or cosmological arguments, which depend on specific assumptions (see, e.g., [78, 114-116]).
} 

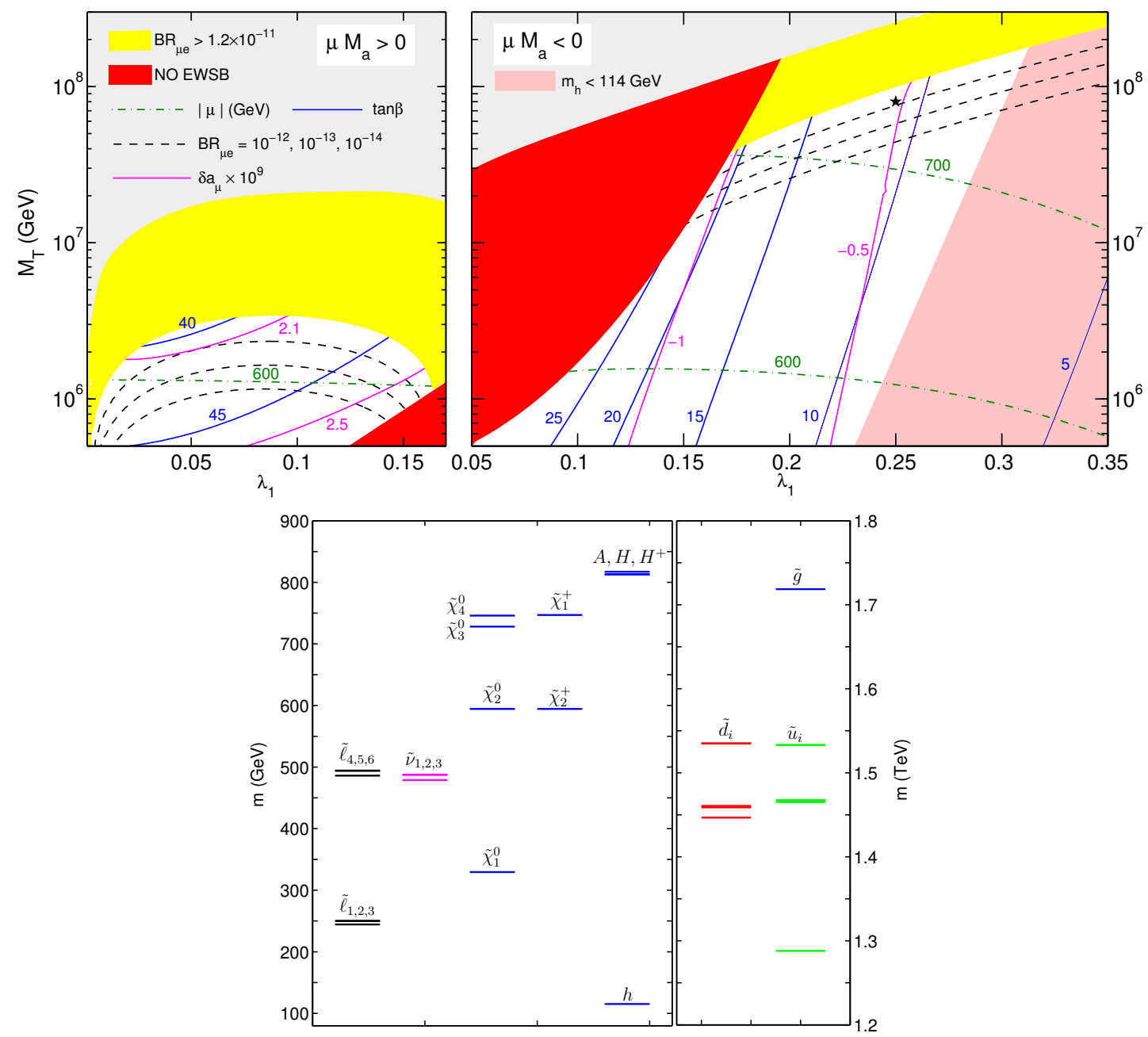

Figure 5. Plots of the $N=4$ model for $B_{T}=60 \mathrm{TeV}$ and normally ordered neutrino spectrum with $0=m_{1}<m_{2} \ll m_{3}$ and $s_{13}=0$. Upper panels: The $\left(\lambda_{1}, M_{T}\right)$ parameter space for $\mu M_{a}>0$ (left) and $\mu M_{a}<0$ (right). The pink region is excluded by the Higgs mass bound $m_{h}>114 \mathrm{GeV}$, the grey one is excluded by perturbativity and the red one by the EWSB conditions. Inside the yellow area $\operatorname{BR}(\mu \rightarrow e \gamma)$ is above the present experimental upper bound. The dashed lines correspond to $\operatorname{BR}(\mu \rightarrow e \gamma)=10^{-12}, 10^{-13}, 10^{-14}$ (from top to bottom.). Isocontours of $\tan \beta$ (solid/blue lines), $|\mu|$ (dash-dotted lines) and $\delta a_{\mu}$ (solid/magenta lines) are also shown. Lower panel: Sparticle and Higgs spectrum for $M_{T}=8 \times 10^{7} \mathrm{GeV}$ and $\lambda_{1}=0.25$. At this point of the parameter space, which is marked by a star $(\star)$ in the upper-right panel, $\tan \beta \simeq 11, \operatorname{BR}(\mu \rightarrow e \gamma) \simeq 1.6 \times 10^{-12}$, $\operatorname{BR}(\tau \rightarrow \mu \gamma) \simeq 6 \times 10^{-10}, \operatorname{BR}(\tau \rightarrow e \gamma) \simeq 2 \times 10^{-13}$.

can become lighter than one or both charginos. This effect is more dramatic in the lower right corner of the $\mu M_{a}>0$ parameter space, where those Higgs masses can decrease even below $200 \mathrm{GeV}$.

The scenario described above can be tested at current and future colliders. In particular, $p p$ collisions at the LHC should produce a significant amount of squark pairs, either directly or through associated squark/gluino production (followed by $\tilde{g} \rightarrow \tilde{q} \bar{q}$ ) [117]. For a spectrum as the one shown in figure 5 the production cross section is about $0.1 \mathrm{pb}$ 
at $\sqrt{s}=14 \mathrm{TeV}$. Once a $\tilde{q}$ is produced, it can decay through well known chains, such as $\tilde{q}_{R} \rightarrow q \tilde{\chi}_{1}^{0} \rightarrow q \tau \tilde{\ell}_{1}, \tilde{q}_{L} \rightarrow q \tilde{\chi}_{2}^{0} \rightarrow q \ell \tilde{\ell} \rightarrow q \ell^{+} \ell^{-} \tilde{\chi}_{1}^{0} \rightarrow q \ell^{+} \ell^{-} \tau \tilde{\ell}_{1}$, or similar ones with charginos and/or sneutrinos (and neutrinos). Hence, in general, the final state of such a $p p$ collision contains SM particles and two NLSPs $\tilde{\ell}_{1}$, which eventually decay to $\tau \tilde{G}$ with rate $\Gamma=m_{\tilde{\ell}_{1}}^{5} /\left(16 \pi F^{2}\right)$. The latter decay can occur either promptly, or at a displaced vertex, or even outside the main detector, as discussed in [109-113, 118, 119]. Let us briefly describe such possibilities in our case, taking into account that $\sqrt{F} \gtrsim \sqrt{\xi_{T} F_{X}}=\sqrt{B_{T} M_{T}}$ and $10^{5} \mathrm{GeV}<\sqrt{B_{T} M_{T}}<10^{7} \mathrm{GeV}$. i) For $\sqrt{F}<10^{7} \mathrm{GeV}$, which includes the case $\sqrt{F} \sim \sqrt{B_{T} M_{T}}$, the NLSP decays occur inside the detector in most of our parameter space, such that the escaping gravitinos contribute to the total missing energy of the event. For instance, if a $p p$ collision produces a pair of $\tilde{q}_{R}$, one can look for the overall signature $p p \rightarrow \tau^{+} \tau^{-} \tau^{+} \tau^{-}+2$ jets $+E_{T}^{\text {miss }}$, possibly with displaced vertices corresponding to the NLSP decays. Instead, if a $\tilde{q}_{L}-\tilde{q}_{R}$ pair is produced, the final state can contain an additional lepton pair $\ell^{+} \ell^{-}$. In both examples, one more jet is present if one of the squarks originates from a gluino. ii) For $\sqrt{F} \gtrsim 10^{7} \mathrm{GeV}$, each NLSP $\tilde{\ell}_{1}$ leaves a track in the main detector and mostly decays outside. In the previous example with a $\tilde{q}_{R}$ pair, the signature would be $p p \rightarrow 2 \tilde{\ell}_{1}+2 \tau+2$ jets. Moreover, in such cases the decay properties of $\tilde{\ell}_{1}$ could be measured by an additional massive detector where $\tilde{\ell}_{1}$ may stop [120-123].

\subsection{Lepton flavour violation}

The experimental signatures mentioned above are also typical of a class of gauge-mediated models, in which flavour is conserved in the SUSY-breaking sector by construction. In contrast, in our scenario LFV is intrinsically present and, therefore, LFV processes are a crucial tool to discriminate our model from pure gauge mediation ones. As already emphasized, the type II mechanism implies that LFV is of minimal type and the basic flavour spurions are $\mathbf{Y}_{T}$ and $\mathbf{Y}_{e}$. Let us focus, for simplicity, on the parameter space where $\tan \beta$ is moderate. Then the leading LFV structure $\mathbf{Y}_{T}^{\dagger} \mathbf{Y}_{T}$, which appears in $\mathbf{m}_{\tilde{L}}^{2}$ (and $\mathbf{A}_{e}$ ), can be related to the neutrino parameters as

$$
\left(\mathbf{m}_{\tilde{L}}^{2}\right)_{i j} \propto B_{T}^{2}\left(\mathbf{Y}_{T}^{\dagger} \mathbf{Y}_{T}\right)_{i j} \propto\left(\frac{M_{T}^{2} \tan ^{2} \beta}{\lambda_{1}}\right)^{2}\left[\mathbf{V}\left(\mathbf{m}_{\nu}^{D}\right)^{2} \mathbf{V}^{\dagger}\right]_{i j} \propto \tan ^{5} \beta M_{T}^{4}\left[\mathbf{V}\left(\mathbf{m}_{\nu}^{D}\right)^{2} \mathbf{V}^{\dagger}\right]_{i j}
$$

where in the last step we have traded the $\lambda_{1}$-dependence for a $\tan \beta$-dependence through the EWSB conditions, i.e. $\lambda_{1}^{2} \sim B_{H} \sim 1 / \tan \beta$ (approximatively valid for $\tan \beta \lesssim 20$ ). Notice that eq. (4.12) includes several approximations, while in the numerical analysis the matching between $\mathbf{Y}_{T}$ and $\mathbf{m}_{\nu}$ proceeds through the effective operators and takes into account RGE effects. However, the latter do not introduce any unknown flavour structure [see eq. (2.4)] and thus the structure of $\mathbf{Y}_{T}$ at the scale $M_{T}$ can be unambiguously predicted (modulo an overall unflavoured factor) once the observable $\mathbf{m}_{\nu}$ is experimentally determined [39-41, 86].

As a consequence of the misalignment between slepton and lepton mass matrices, LFV signals can appear either at high-energy colliders or in low-energy processes. Concerning the former possibility, LFV could show up at the LHC in, e.g., neutralino decays, such as 
$\tilde{\chi}_{2}^{0} \rightarrow \ell_{i}^{ \pm} \tilde{\ell}_{a}^{\mp} \rightarrow \ell_{i}^{ \pm} \ell_{j}^{\mp} \tilde{\chi}_{1}^{0}$ with $i \neq j[83,84,91,124-128]$, or $\tilde{\chi}_{1}^{0} \rightarrow \ell^{ \pm} \tilde{\ell}_{1}^{\mp}$ with $\ell \neq \tau[129]$. Moreover, since LFV also affects lepton-slepton-gravitino couplings [130], a small fraction of NLSP decays $\tilde{\ell}_{1} \rightarrow \ell \tilde{G}$ could produce a lepton $\ell \neq \tau$ [131]. As LFV appears mostly in the left sector in our scenario, the relevant LFV channel in the above examples is expected to be $\tilde{\chi}_{2}^{0} \rightarrow \ell_{i}^{ \pm} \ell_{j}^{\mp} \tilde{\chi}_{1}^{0}$, while LFV effects in the subsequent decays of $\tilde{\chi}_{1}^{0}$ and $\tilde{\ell}_{1}$ are more suppressed since $\tilde{\ell}_{1} \sim \tilde{\tau}_{R}$. For instance, one could look for the LFV decays $\tilde{\chi}_{2}^{0} \rightarrow \mu^{ \pm} \tau^{\mp} \tilde{\chi}_{1}^{0}$ or $\tilde{\chi}_{2}^{0} \rightarrow \mu^{ \pm} e^{\mp} \tilde{\chi}_{1}^{0}$, followed by the flavour-conserving decay $\tilde{\chi}_{1}^{0} \rightarrow \tau^{+} \tau^{-} \tilde{G}$ (or $\tilde{\chi}_{1}^{0} \rightarrow \tilde{\ell}_{1}^{ \pm} \tau^{\mp}$ if the NLSP is long-lived). These options, and perhaps others as well, may deserve further studies.

We now focus on the radiative decays $\ell_{i} \rightarrow \ell_{j} \gamma$ (for which we have already shown some predictions in figure 5), taking them as representative low-energy LFV processes. By using eq. (4.12), we can infer that

$$
\operatorname{BR}\left(\ell_{i} \rightarrow \ell_{j} \gamma\right) \propto\left[\frac{\left(\mathbf{m}_{\tilde{L}}^{2}\right)_{i j}}{\tilde{m}^{4}} \tan \beta\right]^{2} \propto\left(\frac{M_{T}}{B_{T}}\right)^{8}(\tan \beta)^{12}\left[\mathbf{V}\left(\mathbf{m}_{\nu}^{D}\right)^{2} \mathbf{V}^{\dagger}\right]_{i j}
$$

This approximate relation expresses the BRs as a product of a common overall factor (which depends on high powers of $M_{T}, B_{T}$ and $\tan \beta$ ) and a flavour-dependent one, which is determined by the low-energy neutrino parameters only. The latter property is typical of type II models [39-41], while the form of the overall 'unflavoured' factor depends on the specific realization. If we take ratios of BRs, the overall factor drops out and we obtain the following estimates: ${ }^{15}$

$$
\begin{aligned}
& \frac{\operatorname{BR}(\tau \rightarrow \mu \gamma)}{\operatorname{BR}(\mu \rightarrow e \gamma)} \approx\left[\frac{\left(\mathbf{m}_{\tilde{L}}^{2}\right)_{\tau \mu}}{\left(\mathbf{m}_{\tilde{L}}^{2}\right)_{\mu e}}\right]^{2} \frac{\operatorname{BR}\left(\tau \rightarrow \mu \nu_{\tau} \bar{\nu}_{\mu}\right)}{\operatorname{BR}\left(\mu \rightarrow e \nu_{\mu} \bar{\nu}_{e}\right)} \approx \begin{cases}400 & {\left[s_{13}=0\right]} \\
2(3) & {\left[s_{13}=0.2, \delta=0(\pi)\right]}\end{cases} \\
& \frac{\operatorname{BR}(\tau \rightarrow e \gamma)}{\operatorname{BR}(\mu \rightarrow e \gamma)} \approx\left[\frac{\left(\mathbf{m}_{\tilde{L}}^{2}\right)_{\tau e}}{\left(\mathbf{m}_{\tilde{L}}^{2}\right)_{\mu e}}\right]^{2} \frac{\operatorname{BR}\left(\tau \rightarrow e \nu_{\tau} \bar{\nu}_{e}\right)}{\operatorname{BR}\left(\mu \rightarrow e \nu_{\mu} \bar{\nu}_{e}\right)} \approx \begin{cases}0.2 & {\left[s_{13}=0\right]} \\
0.1(0.3) & {\left[s_{13}=0.2, \delta=0(\pi)\right]}\end{cases}
\end{aligned}
$$

We recall that the approximate relations (4.12), (4.13) and (4.14) hold for small or moderate $\tan \beta$. We will not discuss the special features that emerge for large $\tan \beta$, which could be analysed, e.g., along the lines of [86].

Let us now present some numerical examples. In the left panel of figure 6 the three $\mathrm{BR}\left(\ell_{i} \rightarrow \ell_{j} \gamma\right)$ are shown as a function of $\tan \beta$, taking either $B_{T}=60 \mathrm{TeV}$ (solid lines) or $B_{T}=100 \mathrm{TeV}$ (dashed lines), for $M_{T}=8 \times 10^{7} \mathrm{GeV}$ (implying $\mu M_{a}<0$ ), normally

\footnotetext{
${ }^{15}$ We have inserted the best fit values of the neutrino parameters quoted above and assumed a normally ordered neutrino spectrum. The results for inverted ordering are obtained by exchanging $\delta=0 \leftrightarrow \delta=\pi$. Similar results hold when the quartic terms $\left(\mathbf{Y}_{T}^{\dagger} \mathbf{Y}_{T}\right)^{2}$ dominate over the quadratic ones $\mathbf{Y}_{T}^{\dagger} \mathbf{Y}_{T}$ in $\mathbf{m}_{\tilde{L}}^{2}[$ see eq. (4.3)]. The results change if cancellations occur between such terms. A detailed analysis of this case could be performed as in [40, 41], where similar effects are present.
} 

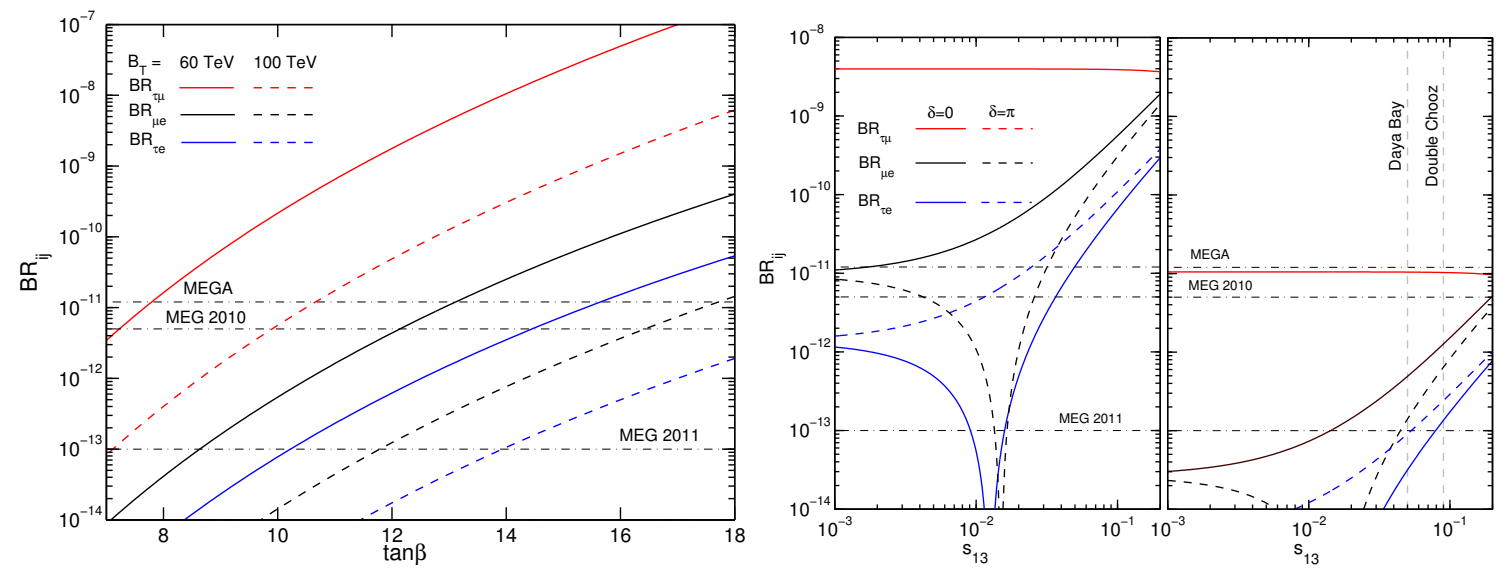

Figure 6. Plots of $\mathrm{BR}\left(\ell_{i} \rightarrow \ell_{j} \gamma\right)$ for $M_{T}=8 \times 10^{7} \mathrm{GeV}$. The present (MEGA) and near-future (MEG) sensitivities on $\mathrm{BR}(\mu \rightarrow e \gamma)$ are also shown. Left panel: The BRs as a function of $\tan \beta$ for $B_{T}=60 \mathrm{TeV}$ (solid lines) or $B_{T}=100 \mathrm{TeV}$ (dashed lines), with $s_{13}=0$. Right panel: The BRs as a function of $s_{13}$ for $B_{T}=60 \mathrm{TeV}$, with $\tan \beta=13$ (8) in the first (second) subpanel. For $\operatorname{BR}(\mu \rightarrow e \gamma)$ and $\operatorname{BR}(\tau \rightarrow e \gamma)$, the solid (dashed) curves correspond to $\delta=0(\pi)$ assuming a normally-ordered neutrino spectrum. We also show the future sensitivity for $s_{13}$ (the RENO expected sensitivity lies between those of Daya Bay and Double Chooz).

ordered neutrino spectrum and $s_{13}=0$. Notice that we have traded $\lambda_{1}$ for $\tan \beta$, which is a more physical and testable parameter. The behaviour of the BRs and their mutual ratios are consistent with the qualitative predictions of eqs. (4.13) and (4.14). For $B_{T}=60 \mathrm{TeV}$, which corresponds to a sparticle spectrum testable at the LHC (see figure 5), $\operatorname{BR}(\mu \rightarrow e \gamma$ ) can be tested at the MEG experiment [106] if $\tan \beta \gtrsim 7$. For $B_{T}=100 \mathrm{TeV}$, gluino and squark masses are pushed above $2 \mathrm{TeV}$, so the discovery of SUSY at the LHC would require more integrated luminosity than in the previous example. In this case LFV decays can still be a valid probe of our scenario, since $\operatorname{BR}(\mu \rightarrow e \gamma)$ could be discovered by MEG for $\tan \beta \gtrsim 10$. Obviously, the ranges of $\tan \beta$ quoted in these examples would change under variations of $M_{T}$ and $B_{T}$, as can be inferred from eq. (4.13) (see also figure 5). Also, from both cases we can see that if $\operatorname{BR}(\mu \rightarrow e \gamma)$ is close to its present bound, $\operatorname{BR}(\tau \rightarrow \mu \gamma)$ is above $10^{-9}$, within the reach of future Super Flavour Factories $[132,133]$. The double panel on the right of figure 6 illustrates the dependence of the BRs on the least known neutrino parameters, namely $s_{13}$ and $\delta$, for $B_{T}=60 \mathrm{TeV}$ and $M_{T}=8 \times 10^{7} \mathrm{GeV}$, with $\tan \beta=13(8)$ in the first (second) subpanel. Regarding $\operatorname{BR}(\mu \rightarrow e \gamma)$ and $\operatorname{BR}(\tau \rightarrow e \gamma)$, the solid (dashed) curves correspond to $\delta=0(\pi)$, while the region between such curves is spanned by intermediate values of $\delta$. The dependence of $\operatorname{BR}(\tau \rightarrow \mu \gamma)$ on $s_{13}$ and $\delta$ is negligible. The first subpanel corresponds to a scenario which could be tested very soon at MEG through the search of $\mu \rightarrow e \gamma$, if $s_{13} \ll 0.01$. Notice that $\operatorname{BR}(\tau \rightarrow \mu \gamma)$ in this example is around $4 \times 10^{-9}$, within the reach of future Super Flavour Factories [132, 133], while $\tau \rightarrow e \gamma$ would be unobservable because $\operatorname{BR}(\tau \rightarrow e \gamma) \sim 10^{-12}$. For $s_{13} \sim 0.01$, $\mathrm{BR}(\mu \rightarrow e \gamma)$ and $\operatorname{BR}(\tau \rightarrow e \gamma)$ can be either enhanced or suppressed since, depending on the value of $\delta$, a cancellation can occur in the LFV quantity $\left[\mathbf{V}\left(\mathbf{m}_{\nu}^{D}\right)^{2} \mathbf{V}^{\dagger}\right]_{i j}[40,41,86]$ (see 
also the third ref. in [95]). The cancellation takes place in $\operatorname{BR}(\mu \rightarrow e \gamma)[\operatorname{BR}(\tau \rightarrow e \gamma)]$ for $\delta=\pi(0)$ in the case of normal ordering, while the opposite occurs for inverted ordering. If $\mathrm{BR}(\mu \rightarrow e \gamma)$ is suppressed by that cancellation mechanism, only $\tau \rightarrow \mu \gamma$ can be observed. In such a case, we can even obtain values of $\operatorname{BR}(\tau \rightarrow \mu \gamma)$ above $10^{-8}$ (i.e., close to its present bound $[103,104])$ by slightly changing the model parameters [see eq. (4.13)]. In the case of partial cancellations, $\mu \rightarrow e \gamma$ could be still probed by MEG for values of $s_{13}$ up to about 0.03, which are in the potential reach of future Neutrino Factories [134]. The second subpanel shows an alternative possibility, in which LFV $\tau$ decays are invisible, whereas $\operatorname{BR}(\mu \rightarrow e \gamma)$ lies in the range $10^{-13}-5 \times 10^{-12}$ if $0.05 \lesssim s_{13}<0.2$. Those values of $\operatorname{BR}(\mu \rightarrow e \gamma)$ should be probed by MEG next year, while the indicated range of $s_{13}$ is within the sensitivity of the present accelerator experiments MINOS [135], OPERA [136] and T2K [137], and the incoming one NOvA [138], as well as of the near-future reactor experiments Double Chooz [139], Daya Bay [140] and RENO [141]. This example shows the importance of the interplay between LFV searches and neutrino oscillation experiments.

For the sake of completeness, we recall that there are good prospects to observe $\mu-e$ LFV also through the processes $\mu \rightarrow e e e$ and $\mu \rightarrow e$ conversion in nuclei, whose rates are correlated to $\mathrm{BR}(\mu \rightarrow e \gamma)$ in our scenario. In particular, dipole-operator dominance implies $\mathrm{CR}(\mu \rightarrow e ; \mathrm{Ti}) \simeq 5 \times 10^{-3} \mathrm{BR}(\mu \rightarrow e \gamma)$ and $\mathrm{CR}(\mu \rightarrow e ; \mathrm{Al}) \simeq 3 \times 10^{-3} \mathrm{BR}(\mu \rightarrow$ e $\gamma)[40,41,142,143]$. Hence, if MEG discovers $\mu \rightarrow e \gamma$ then $\mu \rightarrow e$ conversion could be tested by the dedicated experiments planned at J-PARC [144] and Fermilab [145].

\section{Conclusions}

In the last decade the flurry of experimental neutrino data has provided robust evidence of non-vanishing neutrino masses and mixing angles. This has stimulated further efforts and ideas to understand the origin and pattern of neutrino masses. This issue is part of the wider SM 'flavour problem'. In the case of neutrino masses, one should explain both their flavour structure and their suppression with respect to the charged fermion masses. In this work we have especially addressed the latter aspect in a SUSY framework. At variance with the standard approach, in which neutrino masses effectively arise from the $d=5$, $\Delta L=2$ superpotential operator $\left(H_{2} L\right)^{2} / M$, we have focussed on an interesting alternative mechanism, which relies instead on the $d=6, \Delta L=2$ Kähler operator $\left(H_{1}^{\dagger} L\right)^{2} / M^{2}$, previously proposed in $[37,38]$. We have discussed and further elaborated this idea, first giving a comprehensive model-independent description in an effective-theory approach and then presenting explicit realizations.

In particular, in section 2 we have investigated the above effective operator together with three novel related ones which emerge from SUSY-breaking insertions. In principle, these four $\Delta L=2$ operators have independent coefficients and flavour structures. Two of them contribute to neutrino masses at the tree level and the other two through one-loop corrections at the sparticle threshold. We have also computed the full set of one-loop RGEs, which are required to relate the low-energy effects of those operators with the high-energy scale where they emerge. The effective-theory description we have presented holds both in the MSSM and in simple extensions such as the NMSSM. 
In sections 3 and 4 we have proposed a simple explicit realization of those Kähler operators in a type II seesaw framework, namely by the exchange of heavy $\mathrm{SU}(2)_{W}$-triplet states. The SUSY operator emerges at the tree level, while the origin and the size of the SUSY-breaking ones depend on the mechanism and the scale $M_{S}$ of SUSY-breaking mediation. In particular, the coefficients of the latter operators are related to the SUSYbreaking parameters of the triplet states if $M_{S} \geq M_{T}$, while they can be induced radiatively if $M_{S}<M_{T}$. Finally, we have focussed on the special case $M_{S}=M_{T}$ and proposed a predictive scenario in which the triplets are messengers of both lepton-number violation and SUSY breaking, as in [40, 41].

In the case with $M_{S}=M_{T}$, the MSSM sparticle masses arise by triplet-exchange at the quantum level, via both gauge and Yukawa interactions. In order to generate a mass for the gluino and to preserve perturbative gauge coupling unification, we have embedded the triplets in a messenger sector with coloured states. The free parameters of our model are only three, namely the triplet mass $M_{T}$, the effective SUSY-breaking scale $B_{T}$ and the dimensionless coupling $\lambda_{1}$ (which can be traded for $\tan \beta$ through the EWSB condition). The messenger index $N$ is an additional discrete parameter lying between 4 and 7. Correlations exist among several observables, such as neutrino parameters, sparticle and Higgs masses, and LFV decay rates. A numerical analysis of the parameter space, for the minimal value $N=4$, reveals that the model is phenomenologically viable for $B_{T}>50 \mathrm{TeV}$ and $10^{5} \mathrm{GeV}<M_{T}<10^{9} \mathrm{GeV}$. The latter range is reduced for larger values of $N$, since the lower bound on $M_{T}$ increases.

The MSSM sparticle spectrum is analogous to that of pure gauge mediation models with $N>1$ and not too large mediation scale. The heaviest MSSM sparticle is the gluino, while the lightest one is a stau. The latter, which is in fact the NLSP, can be either short or long-lived and decays into $\tau$ and gravitino. As far as $B_{T} \lesssim 100 \mathrm{TeV}$, the sparticle spectrum can be probed at the LHC (see figure 5) through the production of squarks and gluinos and their subsequent decays into the remaining sparticles.

The presence of LFV allows us to distinguish our scenario from pure gauge mediation models. Such a feature can be tested through the search of either LFV sparticle decays or low-energy LFV processes. We have also emphasized that LFV is of minimal type, as always occurs in type II realizations of the seesaw mechanism. In particular, the flavour structure of the slepton mass matrix is essentially determined by the low-energy neutrino parameters. As a result, the ratios of the $\operatorname{BR}\left(\ell_{i} \rightarrow \ell_{j} \gamma\right)$ can be determined in terms of those parameters, while the absolute values of the BRs depend also on $M_{T}, B_{T}$ and $\tan \beta$. In particular, we can envisage several scenarios for the detection of LFV signals, depending on the yet unknown parameter $s_{13}$. i) If $s_{13} \ll 0.01$ (beyond the planned experimental sensitivity), there are portions of the parameter space in which $\operatorname{BR}(\mu \rightarrow e \gamma)$ and $\operatorname{BR}(\tau \rightarrow \mu \gamma)$ are in the reach of the MEG experiment and Super Flavour Factories, respectively. $i i$ ) If $s_{13} \sim 0.01$ (in the potential reach of future Neutrino Factories) and for suitable values of $\delta$ [i.e. $\delta \sim \pi(0)$ for normal (inverted) ordering in the neutrino spectrum], $\mathrm{BR}(\mu \rightarrow e \gamma)$ is strongly suppressed while $\operatorname{BR}(\tau \rightarrow \mu \gamma)$ can be experimentally accessible. iii) If $s_{13} \gtrsim 0.1$ (reachable by near-future reactor and accelerator neutrino experiments), only $\mu-e \mathrm{LFV}$ can be probed through the measurement of $\operatorname{BR}(\mu \rightarrow e \gamma)$ by the MEG collaboration. 
In conclusion, we have discussed a SUSY scenario which provides an alternative explanation for the smallness of neutrino masses and relates them to sparticle masses in a specific type II realization. Along these lines, further investigations can be envisaged. For instance, on the theoretical side one could address the other aspect of the 'flavour problem', which concerns the origin of the flavour structure of $\mathbf{m}_{\nu}\left(\right.$ or $\left.\mathbf{Y}_{T}\right)$, and explore possible connections with grand unified theories. On the phenomenological side, one could study in more detail the correlation between the sparticle spectrum and the LFV signals, by taking advantage of the interplay between the LHC and low-energy experiments. This would possibly help to discriminate among different mechanisms of SUSY-breaking mediation in the context of the type II seesaw.

Open Access. This article is distributed under the terms of the Creative Commons Attribution Noncommercial License which permits any noncommercial use, distribution, and reproduction in any medium, provided the original author(s) and source are credited.

\section{References}

[1] B.T. Cleveland et al., Measurement of the solar electron neutrino flux with the Homestake chlorine detector, Astrophys. J. 496 (1998) 505 [SPIRES].

[2] Super-Kamkiokande collaboration, J. Hosaka et al., Solar neutrino measurements in Super-Kamiokande-I, Phys. Rev. D 73 (2006) 112001 [hep-ex/0508053] [SPIRES].

[3] Super-Kamiokande collaboration, Y. Ashie et al., A measurement of atmospheric neutrino oscillation parameters by Super-Kamiokande I, Phys. Rev. D 71 (2005) 112005 [hep-ex/0501064] [SPIRES].

[4] SNO collaboration, B. Aharmim et al., Electron energy spectra, fluxes and day-night asymmetries of B-8 solar neutrinos from the 391-day salt phase SNO data set, Phys. Rev. C 72 (2005) 055502 [nucl-ex/0502021] [SPIRES].

[5] SAGE collaboration, J.N. Abdurashitov et al., Measurement of the solar neutrino capture rate with gallium metal. III: Results for the 2002-2007 data-taking period, Phys. Rev. C 80 (2009) 015807 [arXiv:0901.2200] [SPIRES].

[6] K2K collaboration, M.H. Ahn et al., Measurement of neutrino oscillation by the K2K experiment, Phys. Rev. D 74 (2006) 072003 [hep-ex/0606032] [SPIRES].

[7] MINOS collaboration, P. Adamson et al., Measurement of neutrino oscillations with the MINOS detectors in the NuMI beam, Phys. Rev. Lett. 101 (2008) 131802 [arXiv:0806.2237] [SPIRES].

[8] KamLAND collaboration, S. Abe et al., Precision measurement of neutrino oscillation parameters with KamLAND, Phys. Rev. Lett. 100 (2008) 221803 [arXiv:0801.4589] [SPIRES].

[9] CHOOZ collaboration, M. Apollonio et al., Limits on neutrino oscillations from the CHOOZ experiment, Phys. Lett. B 466 (1999) 415 [hep-ex/9907037] [SPIRES].

[10] The MINOS collaboration, P. Adamson et al., New constraints on muon-neutrino to electron-neutrino transitions in MINOS, arXiv:1006.0996 [SPIRES]. 
[11] T. Schwetz, M.A. Tortola and J.W.F. Valle, Three-flavour neutrino oscillation update, New J. Phys. 10 (2008) 113011 [arXiv:0808.2016] [SPIRES].

[12] G.L. Fogli, E. Lisi, A. Marrone, A. Palazzo and A.M. Rotunno, SNO, KamLAND and neutrino oscillations: $\theta_{13}$, arXiv:0905.3549 [SPIRES].

[13] M.C. Gonzalez-Garcia, M. Maltoni and J. Salvado, Updated global fit to three neutrino mixing: status of the hints of $\theta_{13}>0$, JHEP 04 (2010) 056 [arXiv: 1001.4524] [SPIRES].

[14] S. Weinberg, Baryon and lepton nonconserving processes, Phys. Rev. Lett. 43 (1979) 1566 [SPIRES].

[15] P. Minkowski, $\mu \rightarrow e \Gamma$ at a rate of one out of 1-billion muon decays?, Phys. Lett. B 67 (1977) 421 [SPIRES].

[16] M. Gell-Mann, P. Ramond and R. Slansky, in Supergravity, P. Van Nieuwenhuizen and D. Freedman eds., North-Holland, Amsterdam (1979) pg. 315.

[17] T. Yanagida, Horizontal gauge symmetry and masses of neutrinos, in Proceedings of the Workshop on the Unified Theory and the Baryon Number in the Universe, O. Sawada and A. Sugamoto eds., KEK, Tsukuba (1979) pg. 95.

[18] S.L. Glashow, The future of elementary particle physics, in Quarks and Leptons, Cargese France (1979), M. Levy et al. eds., Plenum, New York, U.S.A. (1980) pg. 687.

[19] R.N. Mohapatra and G. Senjanović, Neutrino mass and spontaneous parity nonconservation, Phys. Rev. Lett. 44 (1980) 912 [SPIRES].

[20] J. Schechter and J.W.F. Valle, Neutrino masses in $\mathrm{SU}(2) \times \mathrm{U}(1)$ theories, Phys. Rev. D 22 (1980) 2227 [SPIRES].

[21] M. Magg and C. Wetterich, Neutrino mass problem and gauge hierarchy, Phys. Lett. B 94 (1980) 61 [SPIRES].

[22] G. Lazarides, Q. Shafi and C. Wetterich, Proton lifetime and fermion masses in an $\mathrm{SO}(10)$ model, Nucl. Phys. B 181 (1981) 287 [SPIRES].

[23] R.N. Mohapatra and G. Senjanović, Neutrino masses and mixings in gauge models with spontaneous parity violation, Phys. Rev. D 23 (1981) 165 [SPIRES].

[24] E. Ma and U. Sarkar, Neutrino masses and leptogenesis with heavy Higgs triplets, Phys. Rev. Lett. 80 (1998) 5716 [hep-ph/9802445] [SPIRES].

[25] R. Foot, H. Lew, X.G. He and G.C. Joshi, Seesaw neutrino masses induced by a triplet of leptons, Z. Phys. C 44 (1989) 441 [SPIRES].

[26] E. Ma, Pathways to naturally small neutrino masses, Phys. Rev. Lett. 81 (1998) 1171 [hep-ph/9805219] [SPIRES].

[27] R.N. Mohapatra and J.W.F. Valle, Neutrino mass and baryon-number nonconservation in superstring models, Phys. Rev. D 34 (1986) 1642 [SPIRES].

[28] M.C. Gonzalez-Garcia and J.W.F. Valle, Fast decaying neutrinos and observable flavor violation in a new class of majoron models, Phys. Lett. B 216 (1989) 360 [SPIRES].

[29] A. Zee, A theory of lepton number violation, neutrino majorana mass and oscillation, Phys. Lett. B 93 (1980) 389 [Erratum ibid. B95 (1980) 461] [SPIRES].

[30] K.S. Babu, Model of 'calculable' majorana neutrino masses, Phys. Lett. B 203 (1988) 132 [SPIRES]. 
[31] K.S. Babu and C.N. Leung, Classification of effective neutrino mass operators, Nucl. Phys. B 619 (2001) 667 [hep-ph/0106054] [SPIRES].

[32] A. de Gouvêa and J. Jenkins, A survey of lepton number violation via effective operators, Phys. Rev. D 77 (2008) 013008 [arXiv:0708.1344] [SPIRES].

[33] K.S. Babu, S. Nandi and Z. Tavartkiladze, New mechanism for neutrino mass generation and triply charged higgs bosons at the LHC, Phys. Rev. D 80 (2009) 071702 [arXiv:0905.2710] [SPIRES].

[34] F. Bonnet, D. Hernandez, T. Ota and W. Winter, Neutrino masses from higher than $D=5$ effective operators, JHEP 10 (2009) 076 [arXiv:0907.3143] [SPIRES].

[35] U. Ellwanger, C. Hugonie and A.M. Teixeira, The next-to-minimal supersymmetric standard model, arXiv:0910.1785 [SPIRES].

[36] I. Gogoladze, N. Okada and Q. Shafi, NMSSM and seesaw physics at LHC, Phys. Lett. B 672 (2009) 235 [arXiv:0809.0703] [SPIRES].

[37] J.A. Casas, J.R. Espinosa and I. Navarro, New supersymmetric source of neutrino masses and mixings, Phys. Rev. Lett. 89 (2002) 161801 [hep-ph/0206276] [SPIRES].

[38] J.A. Casas, J.R. Espinosa and I. Navarro, Large mixing angles for neutrinos from infrared fixed points, JHEP 09 (2003) 048 [hep-ph/0306243] [SPIRES].

[39] A. Rossi, Supersymmetric seesaw without singlet neutrinos: Neutrino masses and lepton-flavour violation, Phys. Rev. D 66 (2002) 075003 [hep-ph/0207006] [SPIRES].

[40] F.R. Joaquim and A. Rossi, Gauge and Yukawa mediated supersymmetry breaking in the triplet seesaw scenario, Phys. Rev. Lett. 97 (2006) 181801 [hep-ph/0604083] [SPIRES].

[41] F.R. Joaquim and A. Rossi, Phenomenology of the triplet seesaw mechanism with gauge and Yukawa mediation of SUSY breaking, Nucl. Phys. B 765 (2007) 71 [hep-ph/0607298] [SPIRES].

[42] H.P. Nilles, Supersymmetry, supergravity and particle physics, Phys. Rept. 110 (1984) 1 [SPIRES].

[43] S.P. Martin, A supersymmetry primer, hep-ph/9709356 [SPIRES].

[44] N. Polonsky and S. Su, More corrections to the Higgs mass in supersymmetry, Phys. Lett. B 508 (2001) 103 [hep-ph/0010113] [SPIRES].

[45] A. Brignole, J.A. Casas, J.R. Espinosa and I. Navarro, Low-scale supersymmetry breaking: Effective description, electroweak breaking and phenomenology, Nucl. Phys. B 666 (2003) 105 [hep-ph/0301121] [SPIRES].

[46] M. Dine, N. Seiberg and S. Thomas, Higgs physics as a window beyond the MSSM (BMSSM), Phys. Rev. D 76 (2007) 095004 [arXiv: 0707.0005] [SPIRES].

[47] I. Antoniadis, E. Dudas, D.M. Ghilencea and P. Tziveloglou, MSSM with dimension-five operators $\left(\right.$ MSSM $\left._{5}\right)$, Nucl. Phys. B 808 (2009) 155 [arXiv:0806.3778] [SPIRES].

[48] I. Antoniadis, E. Dudas, D.M. Ghilencea and P. Tziveloglou, MSSM higgs with dimension-six operators, Nucl. Phys. B 831 (2010) 133 [arXiv:0910.1100] [SPIRES].

[49] J.P. Derendinger and C.A. Savoy, Gaugino masses and a new mechanism for proton decay in supersymmetric theories, Phys. Lett. B 118 (1982) 347 [SPIRES].

[50] N. Sakai, Proton decay in models with intermediate scale supersymmetry breaking, Phys. Lett. B 121 (1983) 130 [SPIRES]. 
[51] N. Sakai, Proton decay in locally supersymmetric gut, Nucl. Phys. B 238 (1984) 317 [SPIRES].

[52] Z. Berezhiani, F. Nesti and L. Pilo, Soft SUSY breaking contributions to proton decay, JHEP 10 (2006) 030 [hep-ph/0607303] [SPIRES].

[53] K. Benakli and A.Y. Smirnov, Neutrino-modulino mixing, Phys. Rev. Lett. 79 (1997) 4314 [hep-ph/9703465] [SPIRES].

[54] N. Arkani-Hamed, L.J. Hall, H. Murayama, D. Tucker-Smith and N. Weiner, Small neutrino masses from supersymmetry breaking, Phys. Rev. D 64 (2001) 115011 [hep-ph/0006312] [SPIRES].

[55] F. Borzumati and Y. Nomura, Low-scale see-saw mechanisms for light neutrinos, Phys. Rev. D 64 (2001) 053005 [hep-ph/0007018] [SPIRES].

[56] R.L. Arnowitt, B. Dutta and B. Hu, Yukawa textures, neutrino masses and Hoŕava-Witten M-theory, Nucl. Phys. B 682 (2004) 347 [hep-th/0309033] [SPIRES].

[57] S. Abel, A. Dedes and K. Tamvakis, Naturally small Dirac neutrino masses in supergravity, Phys. Rev. D 71 (2005) 033003 [hep-ph/0402287] [SPIRES].

[58] J. March-Russell and S.M. West, A simple model of neutrino masses from supersymmetry breaking, Phys. Lett. B 593 (2004) 181 [hep-ph/0403067] [SPIRES].

[59] D.A. Demir, L.L. Everett and P. Langacker, Dirac neutrino masses from generalized supersymmetry breaking, Phys. Rev. Lett. 100 (2008) 091804 [arXiv:0712.1341] [SPIRES].

[60] A. Brignole, One-loop Kähler potential in non-renormalizable theories, Nucl. Phys. B 579 (2000) 101 [hep-th/0001121] [SPIRES].

[61] Y. Yamada, Two loop renormalization group equations for soft SUSY breaking scalar interactions: Supergraph method, Phys. Rev. D 50 (1994) 3537 [hep-ph/9401241] [SPIRES].

[62] P.H. Chankowski and Z. Pluciennik, Renormalization group equations for seesaw neutrino masses, Phys. Lett. B 316 (1993) 312 [hep-ph/9306333] [SPIRES].

[63] K.S. Babu, C.N. Leung and J.T. Pantaleone, Renormalization of the neutrino mass operator, Phys. Lett. B 319 (1993) 191 [hep-ph/9309223] [SPIRES].

[64] Y. Grossman and H.E. Haber, Sneutrino mixing phenomena, Phys. Rev. Lett. 78 (1997) 3438 [hep-ph/9702421] [SPIRES].

[65] A. Dedes, H.E. Haber and J. Rosiek, Seesaw mechanism in the sneutrino sector and its consequences, JHEP 11 (2007) 059 [arXiv:0707.3718] [SPIRES].

[66] M. Hirsch, H.V. Klapdor-Kleingrothaus and S.G. Kovalenko, B-L violating masses in softly broken supersymmetry, Phys. Lett. B 398 (1997) 311 [hep-ph/9701253] [SPIRES].

[67] J.M. Frere, M.V. Libanov and S.V. Troitsky, Neutrino masses from nonstandard supersymmetry breaking terms, Phys. Lett. B 479 (2000) 343 [hep-ph/9912204] [SPIRES].

[68] F. Borzumati and A. Masiero, Large muon and electron number violations in supergravity theories, Phys. Rev. Lett. 57 (1986) 961 [SPIRES].

[69] J.-h. Kang, P. Langacker and T.-j. Li, Neutrino masses in supersymmetric $\mathrm{SU}(3)_{C} \times \mathrm{SU}(2)_{L} \times \mathrm{U}(1)_{Y} \times \mathrm{U}(1)$-prime models, Phys. Rev. D 71 (2005) 015012 [hep-ph/0411404] [SPIRES].

[70] M. Dine, W. Fischler and M. Srednicki, Supersymmetric technicolor, Nucl. Phys. B 189 (1981) 575 [SPIRES]. 
[71] S. Dimopoulos and S. Raby, Supercolor, Nucl. Phys. B 192 (1981) 353 [SPIRES].

[72] L. Alvarez-Gaume, M. Claudson and M.B. Wise, Low-energy supersymmetry, Nucl. Phys. B 207 (1982) 96 [SPIRES].

[73] C.R. Nappi and B.A. Ovrut, Supersymmetric extension of the $\mathrm{SU}(3) \times \mathrm{SU}(2) \times \mathrm{U}(1)$ model, Phys. Lett. B 113 (1982) 175 [SPIRES].

[74] S. Dimopoulos and S. Raby, Geometric hierarchy, Nucl. Phys. B 219 (1983) 479 [SPIRES].

[75] M. Dine and A.E. Nelson, Dynamical supersymmetry breaking at low-energies, Phys. Rev. D 48 (1993) 1277 [hep-ph/9303230] [SPIRES].

[76] M. Dine, A.E. Nelson and Y. Shirman, Low-energy dynamical supersymmetry breaking simplified, Phys. Rev. D 51 (1995) 1362 [hep-ph/9408384] [SPIRES].

[77] M. Dine, A.E. Nelson, Y. Nir and Y. Shirman, New tools for low-energy dynamical supersymmetry breaking, Phys. Rev. D 53 (1996) 2658 [hep-ph/9507378] [SPIRES].

[78] G.F. Giudice and R. Rattazzi, Theories with gauge-mediated supersymmetry breaking, Phys. Rept. 322 (1999) 419 [hep-ph/9801271] [SPIRES].

[79] R.N. Mohapatra, N. Okada and H.-B. Yu, Supersymmetry breaking by type II seesaw assisted anomaly mediation, Phys. Rev. D 77 (2008) 115017 [arXiv:0711.0956] [SPIRES].

[80] R.N. Mohapatra, N. Okada and H.-B. Yu, $\nu$-GMSB with type III seesaw and phenomenology, Phys. Rev. D 78 (2008) 075011 [arXiv:0807.4524] [SPIRES].

[81] P. Fileviez Perez, H. Iminniyaz, G. Rodrigo and S. Spinner, Gauge mediated SUSY breaking via seesaw, Phys. Rev. D 81 (2010) 095013 [arXiv:0911.1360] [SPIRES].

[82] A. Melfo, A. Ramirez and G. Senjanović, Type II see-saw dominance in $\mathrm{SO}(10)$, arXiv: 1005.0834 [SPIRES].

[83] M. Hirsch, S. Kaneko and W. Porod, Supersymmetric seesaw type-II: LHC and lepton flavour violating phenomenology, Phys. Rev. D 78 (2008) 093004 [arXiv:0806.3361] [SPIRES].

[84] J.N. Esteves et al., Flavour violation at the LHC: type-I versus type-II seesaw in minimal supergravity, JHEP 05 (2009) 003 [arXiv:0903.1408] [SPIRES].

[85] F. Borzumati and T. Yamashita, Minimal supersymmetric SU(5) model with nonrenormalizable operators: Seesaw mechanism and violation of flavour and CP, arXiv: 0903.2793 [SPIRES].

[86] F.R. Joaquim, Running effects on neutrino parameters and $l_{i} \rightarrow l_{j} \gamma$ predictions in the triplet-extended MSSM, JHEP 06 (2010) 079 [arXiv: 0912.3427] [SPIRES].

[87] S.P. Martin, Generalized messengers of supersymmetry breaking and the sparticle mass spectrum, Phys. Rev. D 55 (1997) 3177 [hep-ph/9608224] [SPIRES].

[88] L. Calibbi, L. Ferretti, A. Romanino and R. Ziegler, Gauge coupling unification, the GUT scale and magic fields, Phys. Lett. B 672 (2009) 152 [arXiv:0812.0342] [SPIRES].

[89] M. Senami and K. Yamamoto, Lepton flavor violation with supersymmetric Higgs triplets in TeV region for neutrino masses and leptogenesis, Phys. Rev. D 69 (2004) 035004 [hep-ph/0305203] [SPIRES].

[90] A. Abada, C. Biggio, F. Bonnet, M.B. Gavela and T. Hambye, Low energy effects of neutrino masses, JHEP 12 (2007) 061 [arXiv:0707.4058] [SPIRES].

[91] M. Raidal et al., Flavour physics of leptons and dipole moments, Eur. Phys. J. C 57 (2008) 13 [arXiv:0801.1826] [SPIRES]. 
[92] G.F. Giudice and R. Rattazzi, Extracting supersymmetry-breaking effects from wave-function renormalization, Nucl. Phys. B 511 (1998) 25 [hep-ph/9706540] [SPIRES].

[93] L.J. Hall and L. Randall, Weak scale effective supersymmetry, Phys. Rev. Lett. 65 (1990) 2939 [SPIRES].

[94] G. D'Ambrosio, G.F. Giudice, G. Isidori and A. Strumia, Minimal flavour violation: An effective field theory approach, Nucl. Phys. B 645 (2002) 155 [hep-ph/0207036] [SPIRES].

[95] V. Cirigliano, B. Grinstein, G. Isidori and M.B. Wise, Minimal flavor violation in the lepton sector, Nucl. Phys. B 728 (2005) 121 [hep-ph/0507001] [SPIRES].

[96] S. Davidson and F. Palorini, Various definitions of minimal flavour violation for leptons, Phys. Lett. B 642 (2006) 72 [hep-ph/0607329] [SPIRES].

[97] K.S. Babu and C. Kolda, Higgs-mediated $\tau \rightarrow 3 \mu$ in the supersymmetric seesaw model, Phys. Rev. Lett. 89 (2002) 241802 [hep-ph/0206310] [SPIRES].

[98] A. Dedes, J.R. Ellis and M. Raidal, Higgs mediated $B_{s, d}^{0} \rightarrow \mu \tau$, e $\tau$ and $\tau \rightarrow 3 \mu$, e $\mu \mu$ decays in supersymmetric seesaw models, Phys. Lett. B 549 (2002) 159 [hep-ph/0209207] [SPIRES].

[99] A. Brignole and A. Rossi, Lepton flavour violating decays of supersymmetric Higgs bosons, Phys. Lett. B 566 (2003) 217 [hep-ph/0304081] [SPIRES].

[100] A. Brignole and A. Rossi, Anatomy and phenomenology of mu tau lepton flavour violation in the MSSM, Nucl. Phys. B 701 (2004) 3 [hep-ph/0404211] [SPIRES].

[101] LEP Working Group for Higgs boson searches collaboration, R. Barate et al., Search for the standard model Higgs boson at LEP, Phys. Lett. B 565 (2003) 61 [hep-ex/0306033] [SPIRES].

[102] MEGA collaboration, M.L. Brooks et al., New limit for the family-number non-conserving decay $\mu^{+} \rightarrow e_{\gamma}^{+}$, Phys. Rev. Lett. 83 (1999) 1521 [hep-ex/9905013] [SPIRES].

[103] BABAR collaboration, B. Aubert et al., Searches for lepton flavor violation in the decays $\tau^{ \pm} \rightarrow e^{ \pm} \gamma$ and $\tau^{ \pm} \rightarrow \mu^{ \pm} \gamma$, Phys. Rev. Lett. 104 (2010) 021802 [arXiv:0908.2381] [SPIRES].

[104] Belle collaboration, K. Hayasaka et al., New search for $\tau \rightarrow \mu \gamma$ and $\tau \rightarrow e \gamma$ decays at Belle, Phys. Lett. B 666 (2008) 16 [arXiv:0705.0650] [SPIRES].

[105] S. Heinemeyer, W. Hollik and G. Weiglein, FeynHiggs: a program for the calculation of the masses of the neutral CP-even Higgs bosons in the MSSM, Comput. Phys. Commun. 124 (2000) 76 [hep-ph/9812320] [SPIRES].

[106] MEG collaboration, E. Baracchini, First result from the MEG experiment, arXiv: 1005. 2569 [SPIRES].

[107] M. Davier et al., The discrepancy between $\tau$ and $e^{+} e^{-}$spectral functions revisited and the consequences for the muon magnetic anomaly, Eur. Phys. J. C 66 (2010) 127 [arXiv: 0906 .5443] [SPIRES].

[108] M. Davier, A. Hoecker, B. Malaescu, C.Z. Yuan and Z. Zhang, Reevaluation of the hadronic contribution to the muon magnetic anomaly using new $e^{+} e^{-} \rightarrow \pi^{+} \pi^{-}$cross section data from BABAR, Eur. Phys. J. C 66 (2010) 1 [arXiv:0908.4300] [SPIRES].

[109] S. Dimopoulos, M. Dine, S. Raby and S.D. Thomas, Experimental signatures of low energy gauge mediated supersymmetry breaking, Phys. Rev. Lett. 76 (1996) 3494 [hep-ph/9601367] [SPIRES]. 
[110] S. Dimopoulos, S.D. Thomas and J.D. Wells, Sparticle spectroscopy and electroweak symmetry breaking with gauge-mediated supersymmetry breaking,

Nucl. Phys. B 488 (1997) 39 [hep-ph/9609434] [SPIRES].

[111] J.A. Bagger, K.T. Matchev, D.M. Pierce and R.-j. Zhang, Weak-scale phenomenology in models with gauge-mediated supersymmetry breaking, Phys. Rev. D 55 (1997) 3188 [hep-ph/9609444] [SPIRES].

[112] S. Ambrosanio, G.D. Kribs and S.P. Martin, Signals for gauge-mediated supersymmetry breaking models at the CERN LEP2 collider, Phys. Rev. D 56 (1997) 1761 [hep-ph/9703211] [SPIRES].

[113] J.L. Feng and T. Moroi, Tevatron signatures of longlived charged sleptons in gauge mediated supersymmetry breaking models, Phys. Rev. D 58 (1998) 035001 [hep-ph/9712499] [SPIRES].

[114] J.A. Grifols, R.N. Mohapatra and A. Riotto, New astrophysical constraints on the mass of the superlight gravitino, Phys. Lett. B 400 (1997) 124 [hep-ph/9612253] [SPIRES].

[115] M. Viel, J. Lesgourgues, M.G. Haehnelt, S. Matarrese and A. Riotto, Constraining warm dark matter candidates including sterile neutrinos and light gravitinos with WMAP and the Lyman- alpha forest, Phys. Rev. D 71 (2005) 063534 [astro-ph/0501562] [SPIRES].

[116] J.L. Feng, M. Kamionkowski and S.K. Lee, Light gravitinos at colliders and implications for cosmology, Phys. Rev. D 82 (2010) 015012 [arXiv: 1004.4213] [SPIRES].

[117] W. Beenakker, R. Hopker, M. Spira and P.M. Zerwas, Squark and gluino production at hadron colliders, Nucl. Phys. B 492 (1997) 51 [hep-ph/9610490] [SPIRES].

[118] I. Hinchliffe and F.E. Paige, Measurements in gauge mediated SUSY breaking models at LHC, Phys. Rev. D 60 (1999) 095002 [hep-ph/9812233] [SPIRES].

[119] S. Ambrosanio, B. Mele, S. Petrarca, G. Polesello and A. Rimoldi, Measuring the SUSY breaking scale at the LHC in the slepton NLSP scenario of GMSB models, JHEP 01 (2001) 014 [hep-ph/0010081] [SPIRES].

[120] W. Buchmüller, K. Hamaguchi, M. Ratz and T. Yanagida, Supergravity at colliders, Phys. Lett. B 588 (2004) 90 [hep-ph/0402179] [SPIRES].

[121] K. Hamaguchi, Y. Kuno, T. Nakaya and M.M. Nojiri, A study of late decaying charged particles at future colliders, Phys. Rev. D 70 (2004) 115007 [hep-ph/0409248] [SPIRES].

[122] J.L. Feng and B.T. Smith, Slepton trapping at the large hadron and international linear colliders, Phys. Rev. D 71 (2005) 015004 [Erratum ibid. D 71 (2005) 019904] [hep-ph/0409278] [SPIRES].

[123] K. Hamaguchi, M.M. Nojiri and A. de Roeck, Prospects to study a long-lived charged next lightest supersymmetric particle at the LHC, JHEP 03 (2007) 046 [hep-ph/0612060] [SPIRES].

[124] K. Agashe and M. Graesser, Signals of supersymmetric lepton flavor violation at the LHC, Phys. Rev. D 61 (2000) 075008 [hep-ph/9904422] [SPIRES].

[125] I. Hinchliffe and F.E. Paige, Lepton flavor violation at the LHC, Phys. Rev. D 63 (2001) 115006 [hep-ph/0010086] [SPIRES].

[126] J. Hisano, R. Kitano and M.M. Nojiri, Slepton oscillation at large hadron collider, Phys. Rev. D 65 (2002) 116002 [hep-ph/0202129] [SPIRES]. 
[127] D.F. Carvalho, J.R. Ellis, M.E. Gomez, S. Lola and J.C. Romao, $\tau$ flavour violation in sparticle decays at the LHC, Phys. Lett. B 618 (2005) 162 [hep-ph/0206148] [SPIRES].

[128] A. Bartl et al., Test of lepton flavour violation at LHC, Eur. Phys. J. C 46 (2006) 783 [hep-ph/0510074] [SPIRES].

[129] R. Kitano, A clean slepton mixing signal at the LHC, JHEP 03 (2008) 023 [arXiv:0801.3486] [SPIRES].

[130] A. Brignole and A. Rossi, Flavour non-conservation in goldstino interactions, Nucl. Phys. B 587 (2000) 3 [hep-ph/0006036] [SPIRES].

[131] K. Hamaguchi and A. Ibarra, Probing lepton flavour violation in slepton NLSP scenarios, JHEP 02 (2005) 028 [hep-ph/0412229] [SPIRES].

[132] T. Aushev et al., Physics at super B factory, arXiv:1002.5012 [SPIRES].

[133] M. Bona et al., SuperB: a high-luminosity asymmetric $e^{+} e^{-}$super flavor factory. Conceptual design report, arXiv:0709.0451 [SPIRES].

[134] ISS Physics Working Group collaboration, A. Bandyopadhyay et al., Physics at a future neutrino factory and super-beam facility, Rept. Prog. Phys. 72 (2009) 106201 [arXiv:0710.4947] [SPIRES].

[135] MINOS collaboration, J.M. Paley, Recent results and future prospects from MINOS, arXiv:0901.2131 [SPIRES].

[136] M. Komatsu, P. Migliozzi and F. Terranova, Sensitivity to $\Theta_{13}$ of the CERN to Gran Sasso neutrino beam, J. Phys. G 29 (2003) 443 [hep-ph/0210043] [SPIRES].

[137] T2K collaboration, Y. Obayashi, Reach of future accelerator and reactor neutrino efforts, arXiv:0807.4012 [SPIRES].

[138] NOvA collaboration, D.S. Ayres et al., NOvA proposal to build a 30-kiloton off-axis detector to study neutrino oscillations in the Fermilab NuMI beamline, hep-ex/0503053 [SPIRES].

[139] Double CHOOZ collaboration, F. Ardellier et al., Double CHOOZ: A search for the neutrino mixing angle $\theta_{13}$, hep-ex/0606025 [SPIRES].

[140] DaYA-BAY collaboration, X. Guo et al., A precision measurement of the neutrino mixing angle $\theta_{13}$ using reactor antineutrinos at Daya Bay, hep-ex/0701029 [SPIRES].

[141] RENO collaboration, S.-B. Kim, RENO for neutrino mixing angle $\Theta_{13}$, Prog. Part. Nucl. Phys. 64 (2010) 346 [SPIRES].

[142] J. Hisano, T. Moroi, K. Tobe and M. Yamaguchi, Lepton-flavor violation via right-handed neutrino Yukawa couplings in supersymmetric standard model, Phys. Rev. D 53 (1996) 2442 [hep-ph/9510309] [SPIRES].

[143] R. Kitano, M. Koike and Y. Okada, Detailed calculation of lepton flavor violating muon electron conversion rate for various nuclei, Phys. Rev. D 66 (2002) 096002 [Erratum ibid. D 76 (2007) 059902] [hep-ph/0203110] [SPIRES].

[144] COMET collaboration, Y.G. Cui et al., Conceptual design report for experimental search for lepton flavor violating $\mu^{-}-e^{-}$conversion at sensitivity of $10^{-16}$ with a slow-extracted bunched proton beam (COMET), KEK-2009-10 http://ccdb4fs.kek.jp/tiff/2009/0924/0924011.pdf [SPIRES].

[145] Mu2E collaboration, R.M. Carey et al., Proposal to search for $\mu^{-} N \rightarrow e^{-} N$ with a single event sensitivity below $10^{-16}$, FERMILAB-PROPOSAL-0973 [SPIRES]. 